Federal Reserve Bank of Minneapolis

Research Department Staff Report 432

Revised February 2012

\title{
Consumption and Labor Supply with Partial Insurance: An Analytical Framework*
}

\author{
Jonathan Heathcote \\ Federal Reserve Bank of Minneapolis \\ and CEPR \\ heathcote@minneapolisfed.org \\ Kjetil Storesletten \\ Federal Reserve Bank of Minneapolis, \\ University of Oslo, \\ and CEPR \\ kjstore@econ.uio.no
}

Giovanni L. Violante

New York University, CEPR, and NBER

glv2@nyu.edu

\section{ABSTRACT}

This paper develops a model with partial insurance against idiosyncratic wage shocks to quantify risk sharing, and to decompose inequality into life-cycle shocks versus initial heterogeneity in preferences and productivity. Closed-form solutions are obtained for equilibrium allocations and for moments of the joint distribution of consumption, hours, and wages. We prove identification and estimate the model with data from the CEX and the PSID over the period 1967-2006. We find that (i) $40 \%$ of permanent wage shocks pass through to consumption; (ii) the share of wage risk insured privately increased until the early 1980s and remained stable thereafter; (iii) life-cycle productivity shocks account for half of the cross-sectional variance of wages and earnings, but for much less of dispersion in consumption or hours worked.

*Heathcote and Violante's research is supported by a grant from the National Science Foundation (SES 0418029). Storesletten thanks the Research Council of Norway and the Centre of Equality, Social Organization, and Performance. The views expressed herein are those of the authors and not necessarily those of the Federal Reserve Bank of Minneapolis or the Federal Reserve System. 


\section{Introduction}

How effectively can households smooth idiosyncratic wage fluctuations via private insurance arrangements and labor supply adjustments? To what degree has the four-decade-long rise in US wage dispersion passed through to inequality in consumption and hours worked? More broadly, what is the role of uninsurable life-cycle shocks to wages relative to initial heterogeneity in skills and preferences in accounting for observed inequality? The purpose of this paper is to measure the degree of risk sharing achieved by US households, and to examine how it has changed over the past forty years. Quantifying the extent of private risk sharing is important in determining the appropriate role for redistributive taxation and public insurance programs.

The measurement of risk sharing has been approached in two ways in the existing literature. The first approach is to build a structural equilibrium model, and to use it as an artificial laboratory to study the response of consumption to individual income fluctuations. One prominent example is the standard incomplete-markets model, where households self-insure against income fluctuations by borrowing and lending via a risk-free bond (see Heathcote, Storesletten, and Violante (2009) for a survey). The key limitation of the structural approach is that the amount of risk sharing in the model is always sensitive to the model assumptions regarding the asset market structure and the insurance channels available to agents.

The alternative approach in the literature is to quantify the overall degree of insurability of income innovations, while remaining somewhat agnostic about the specific sources of risk sharing. Deaton (1997) pioneered this methodology. ${ }^{1}$ An important recent example of this less structural approach to measuring insurance is Blundell, Pistaferri, and Preston (2008), who use longitudinal data on income and consumption (constructed through an imputation procedure) to estimate the degree to which permanent changes in earnings pass through to household consumption. This methodology requires a long panel of high-quality income

\footnotetext{
${ }^{1}$ Deaton (1997, pp. 372-374) writes: "Saving is only one of the ways people can protect their consumption against fluctuations in their income. An alternative is to rely on other people, to share risk with friends and kin, with neighbors, or with other anonymous participants through private or government insurance schemes, or through participation in financial markets ... $[\mathrm{T}]$ he very multiplicity of existing mechanisms makes it likely that there is at least partial insurance through financial or social institutions, and that such risk sharing adds to the possibilities for autarkic consumption smoothing through intertemporal transfers of money or goods ... Although it is possible to examine the mechanisms, the insurance contracts, tithes and transfers, their multiplicity makes it attractive to look directly at the magnitude that is supposed to be smoothed, namely consumption."
} 
and consumption data. Moreover, the estimation procedure embeds assumptions on the equilibrium process for consumption, and pass-through coefficients will be biased if these assumptions are not satisfied (see Kaplan and Violante (2010) for a discussion).

This paper pursues a new approach to measuring risk sharing that combines elements from both these traditions. We estimate the overall amount of private consumption insurance in the context of a structural equilibrium model that allows for a flexible financial market structure - and thus does not "hardwire" agents' access to insurance. Our framework is an augmented version of the standard incomplete-markets model with idiosyncratic wage risk. We assume isoelastic preferences over consumption and hours worked, and allow for heterogeneity in the taste for work. Wages have two orthogonal stochastic components, which we label (privately) "insurable" and "uninsurable," respectively. Agents can perfectly smooth the "insurable" component. The "uninsurable" component can only be smoothed via adjustments to own hours worked, via government redistribution, or through borrowing and lending in a riskless bond.

The equilibrium allocations of consumption and hours emerging from our framework can be expressed in closed form, as log-linear functions of the idiosyncratic distaste for work and of the two wage components. An important step toward characterizing allocations is to show that in equilibrium the bond is not used to smooth "uninsurable" shocks - a result that extends the logic in Constantinides and Duffie (1996) to a much richer environment. The analytical derivation of allocations is the first theoretical contribution of the paper.

These closed-form log-linear allocations make it possible to compute and interpret crosssectional variances and covariances of the joint equilibrium distribution of wages, hours, and consumption. We use information contained in both the "macro facts" on the distributions of these variables in levels that have motivated recent macroeconomic investigations (e.g., Attanasio and Davis, 1996; Krueger and Perri, 2006; Heathcote, Storesletten, and Violante 2010b), and the "micro facts" on the distributions in growth rates that have been the primary focus of labor economists (e.g., Abowd and Card, 1989; Blundell, Pistaferri, and Preston, 2008). The analytical expressions for these cross-sectional moments allow us to formally prove identification of all the model's parameters given standard micro data sets - something that is usually impossible in large scale structural models. Proof of identification is our second theoretical contribution.

Most of the literature on risk sharing focuses on how labor market shocks transmit to consumption (see Jappelli and Pistaferri (2010) for a recent survey). We argue that data on 
labor supply are also informative about the degree of insurance, because theory predicts that households should adjust hours more strongly in response to insurable versus uninsurable wage increases, reflecting the absence of offsetting wealth effects in the former case. We prove that the model is in fact fully identified given only panel data on wages and hours worked, i.e., without any consumption data. Given uncertainty about the quality of self-reported consumption expenditures (e.g., Attanasio, Battistin, and Ichimura, 2007; Aguiar and Bils, 2011), it is useful to be able to estimate insurance without using consumption data, and to assess whether estimates of insurance derived from wage and hours data alone are consistent with those that use consumption moments. Exploiting moments involving hours worked to quantify risk sharing is our third contribution.

We estimate the model using data on wages and hours from the Panel Study of Income Dynamics (PSID) over the period 1967-2006, and consumption data from the Consumer Expenditure Survey (CEX) over the period 1980-2006. The estimated model replicates well the evolution of the empirical cross-sectional distribution over wages, hours worked and consumption, both over time and over the life cycle. The estimated value for the intertemporal elasticity of substitution for consumption is $1 / 1.56$, while the Frisch elasticity of labor supply with respect to pre-tax wages is 0.38 . We estimate significant heterogeneity in the distaste for work, which is needed to account for the observed joint distribution over consumption and hours worked. The estimation also recovers time series for the insurable and uninsurable fractions of wage dispersion, which allow us to address the three questions that motivate our investigation.

First, we ask how much individual wage risk can be smoothed, and what are the relative contributions to smoothing of private risk sharing, government-provided insurance, and labor supply adjustments. Blundell, Pistaferri, and Preston (2008) argue that a natural way to measure consumption insurance is to quantify how much of a typical permanent shock to wages passes through to consumption. Our model suggests that this pass-through coefficient is around $40 \%$, or equivalently that $60 \%$ of permanent wage fluctuations are effectively smoothed. Where does this insurance come from? Half reflects private risk sharing, one-third reflects progressive taxation, and the rest reflects adjustments to labor supply. A caveat is in order. A common alternative metric for consumption smoothing in the literature is the ratio of the within-cohort change in the variance of log consumption to the corresponding change in the variance of log income (e.g., Blundell and Preston, 1998; Storesletten, Telmer, and Yaron, 2004a). In our framework the two measures of insurance coincide only when pro- 
gressive taxation and labor supply are absent as insurance channels. Our baseline parameter estimates indicate a significantly smaller value for the ratio-of-variances statistic than for the pass-through coefficient.

Second, we ask how risk sharing has changed over time. We find that US households were effectively able to insure two-thirds of the sharp increase in wage inequality over the past 40 years. Private risk sharing increased during the 1970s: in 1967 the insurable component of wages accounted for around $25 \%$ of the cross-sectional variance of log wages, while by the early 1980s this fraction was around 45\%. Since then, the variances of the insurable and uninsurable components of wages have risen at a similar rate, leaving the fraction of wage fluctuations insured relatively stable. Data on hours worked are an essential input for these estimates, since no consumption data in available prior to 1980, and it is the observed increase in the covariance between wages and hours that indicates an increase in the degree of risk sharing. Reassuringly, after 1980, data on hours worked and data on consumption deliver consistent estimates for the relative importance of insurable and uninsurable shocks.

Third, we use the estimated model to decompose inequality in the cross section into components reflecting life-cycle shocks versus initial heterogeneity in productivity and the disutility of work effort. This decomposition is unique and additive in our framework. Roughly half of the total cross-sectional variance in wages and earnings reflects life-cycle shocks to wages. In contrast, these shocks account for less than $20 \%$ of the cross-sectional variances of consumption and hours worked. Net of measurement error, the most important source of dispersion in consumption is initial heterogeneity in productivity. For hours worked, in contrast, it is heterogeneity in preferences.

The rest of the paper is organized as follows. Section 2 develops our framework, derives the equilibrium allocations, and explains how we obtain tractability. In Section 3, we derive closed-form expressions for the equilibrium cross-sectional moments. Section 4 proves how these moments allow us to identify all the structural parameters of the model, and describes the data and estimation algorithm. Section 5 lays out the results of the quantitative analysis. Section 6 concludes.

\section{Model economy}

We first describe the model formally. Next, we discuss the key assumptions in detail.

Demographics We adopt the Yaari perpetual youth model: agents are born at age zero 
and survive from age $a$ to age $a+1$ with constant probability $\delta<1$. A new generation with mass $(1-\delta)$ enters the economy at each date $t$. Thus, the measure of agents of age $a$ is $(1-\delta) \delta^{a}$, and the total population size is unity.

Preferences Lifetime utility for an agent born (i.e., entering the labor market) in cohort birth year $b$ is given by

$$
\mathbb{E}_{b} \sum_{t=b}^{\infty}(\beta \delta)^{t-b} u\left(c_{t}, h_{t} ; \varphi\right),
$$

where the expectation is taken over sequences of shocks defined below. Here $c_{t}$ denotes consumption at date $t$ for an agent of age $a=t-b$, while $h_{t}$ is the corresponding value for hours worked. Agents discount the future at rate $\beta \delta$, where $\beta<1$ is the pure discount factor. Period utility is

$$
u\left(c_{t}, h_{t} ; \varphi\right)=\frac{c_{t}^{1-\gamma}-1}{1-\gamma}-\exp (\varphi) \frac{h_{t}^{1+\sigma}}{1+\sigma}
$$

The parameter $\gamma$ is the inverse of the intertemporal elasticity of substitution for consumption, and $\sigma$ governs the elasticity of labor supply. ${ }^{2}$ The preference weight $\varphi$ captures the strength of an individual's aversion to work. The distribution of $\varphi$ for the cohort with birth year $t$ is denoted $F_{\varphi t}$, with cohort-specific variance $v_{\varphi t}$. We incorporate preference heterogeneity because, as we will show, it is important for explaining the observed crosssectional joint distribution over wages, hours, and consumption. ${ }^{3}$ In Section 6 we discuss how our results extend to alternative preference specifications.

Idiosyncratic risk The population in the economy is partitioned into groups that we will refer to as "islands," where each island contains a continuum of individual agents. Agents face labor productivity shocks at the individual level, which are uncorrelated across members of each island, and shocks at the island level, which are common to all members of a given island, but uncorrelated across islands. Individual labor productivity is denoted $w$ and given (in logs) by the sum of the island-level component, denoted $\alpha$, and the (orthogonal) individual-level component, denoted $\varepsilon$ :

$$
\log w_{t}=\alpha_{t}+\varepsilon_{t}
$$

\footnotetext{
${ }^{2}$ The parameter $\gamma$ is also related to risk aversion. In particular, the coefficient of relative risk aversion is $1 /(1 / \gamma+1 / \sigma)$ (see Swanson, forthcoming). As we explain below, the most important role of $\gamma$ in our model is that it determines the relative strength of income and substitution effects on hours worked.

${ }^{3}$ It has long been recognized that a sizeable fraction of cross-sectional dispersion in hours worked is unrelated to dispersion in wages (e.g., Abowd and Card, 1989).
} 
The market structure outlined below will assume differential trading opportunities between versus within islands, translating into differential insurance against shocks to $\alpha$ versus $\varepsilon$.

The island-level component $\alpha$ follows a random walk:

$$
\alpha_{t}=\alpha_{t-1}+\omega_{t}
$$

where the innovation $\omega$ is drawn from the distribution $F_{\omega t}$ with variance $v_{\omega t}$ at time $t$. The individual-level component $\varepsilon$ is itself the sum of two orthogonal random variables:

$$
\varepsilon_{t}=\kappa_{t}+\theta_{t}
$$

Here $\theta$ is a transitory (independently distributed over time) shock drawn from $F_{\theta t}$ with variance $v_{\theta t}$, while $\kappa$ is a permanent component that follows a second unit root process:

$$
\kappa_{t}=\kappa_{t-1}+\eta_{t}
$$

where the innovation $\eta$ is drawn from the distribution $F_{\eta t}$ with variance $v_{\eta t}{ }^{4}$

Agents who enter the labor market at age $a=0$ in year $t$ draw initial realizations $\alpha^{0}$ and $\kappa^{0}$ from distributions $F_{\alpha^{0} t}$ and $F_{\kappa^{0} t}$, with cohort-specific variances $v_{\alpha^{0} t}$ and $v_{\kappa^{0} t}$. The initial draws $\varphi, \alpha^{0}$, and $\kappa^{0}$ are assumed to be uncorrelated. ${ }^{5}$

A law of large numbers (e.g., Uhlig, 1996) can be applied twice so that individual-level $\varepsilon$ shocks wash out within an island, and island-level $\alpha$ shocks induce no aggregate uncertainty in the economy as a whole (see Attanasio and Ríos-Rull (2000) for a similar structure).

Production Production of the final consumption good takes place through a constant returns to scale technology with labor as the only input. The economy-wide good and labor markets are perfectly competitive. Hence, individual wages equal individual productivities (units of effective labor per hour worked).

Taxes and redistribution The government operates a progressive tax-transfer system that provides public insurance. Following Benabou (2002), an individual with a gross labor

\footnotetext{
${ }^{4}$ The assumed statistical process for individual efficiency units - unit root plus i.i.d. shocks - has a long tradition in the literature that estimates statistical models for individual wage dynamics (see, e.g., MaCurdy, 1982). The empirical autocovariance function for individual wages displays a sharp decline at the first lag, indicating the presence of a transitory component in wages. At the same time, within-cohort wage dispersion increases approximately linearly with age, suggesting the presence of permanent shocks.

${ }^{5}$ The initial draws $\left(\varphi, \alpha^{0}, \kappa^{0}\right)$ could in principle be correlated if, for example, wages at labor market entry are a function of schooling, and schooling depends on the preference weight, $\varphi$. In a previous version of this paper, we allowed for correlation between $\alpha^{0}$ and $\varphi$, but the estimated correlation coefficient was insignificantly different from zero.
} 
income $y_{t}=w_{t} h_{t}$ receives disposable post-government earnings $\tilde{y}_{t}$ given by

$$
\tilde{y}_{t}=\lambda\left(y_{t}\right)^{1-\tau} .
$$

The fiscal parameters $\lambda$ and $\tau$ are assumed constant over time. Loosely speaking, $\lambda$ defines the level of taxation, while $\tau \geq 0$ defines the rate of progressivity built into the tax and transfer system. To see this, note that $\log \left(\tilde{y}_{t}\right)=\log (\lambda)+(1-\tau) \log \left(y_{t}\right)$, and thus $(1-\tau)$ defines the elasticity of after-tax earnings to pre-tax earnings. For $\tau=0$ the system implies a flat tax $1-\lambda$ on labor income, while for $\tau>0$ the tax and transfer system is progressive. The government uses aggregate net tax revenue to finance a non-valued public consumption good $G_{t}$, which adjusts to balance the government budget on a period-by-period basis.

While this model of taxation and transfers is simple, it is sufficiently flexible to offer a reasonable approximation to the actual US tax and transfer system (see Section 4.3).

Market structure All assets in the economy are in zero net supply, and asset markets are competitive. At birth, each agent is endowed with zero financial wealth. ${ }^{6}$ Individuals born in year $b$ draw values for $\alpha^{0}$ and $\varphi$ before any markets open. They are then allocated to an island, which is defined by an ex ante unknown sequence $\left\{\omega_{t}\right\}_{t=b+1}^{\infty}$ that will apply to all island members. Within each island, agents trade a complete set of insurance contracts. In particular, in every period $t \geq b$, agents can purchase contracts indexed to $s_{t+1}=\left(\omega_{t+1}, \eta_{t+1}, \theta_{t+1}\right){ }^{7}$ Scope for insurance across islands is more limited: agents can only trade insurance contracts indexed to their individual-level shocks $\left(\eta_{t+1}, \theta_{t+1}\right)$, but inter-island contracts contingent on the realization of the island-level shock $\omega_{t+1}$ are ruled out.

Insurance contracts incorporate mortality risk: if an agent purchases one unit of insurance against any state $s_{t+1}$, the contract pays $\delta^{-1}$ units of consumption if the agent survives to the next period and $s_{t+1}$ is realized, and 0 units otherwise.

Note that agents can effectively trade risk-free bonds freely within or across islands. In particular, purchasing $\delta$ units of insurance for every possible realization of the pair $\left(\eta_{t+1}, \theta_{t+1}\right)$ delivers one unit of consumption risk-free in the next period.

Information Agents are assumed to take as given the sequences of distributions $\left\{F_{\varphi t}, F_{\alpha^{0} t}\right.$, $\left.F_{\kappa^{0} t}, F_{\omega t}, F_{\eta t}, F_{\theta t}\right\}$. Thus they have perfect foresight over future wage distributions. ${ }^{8}$

\footnotetext{
${ }^{6}$ It is straightforward to relax the assumption of zero initial individual financial wealth. The key requirement, as will become clear below, is that average initial wealth on each island is zero.

${ }^{7}$ New labor market entrants can also purchase contracts indexed to $\left(\kappa^{0}, \theta_{b}\right)$.

${ }^{8}$ This assumption is not required for tractability. Alternatively, one could assume that the variances of these distributions themselves follow some stochastic process. The expression for the equilibrium interest rate would be affected, but equilibrium allocations would remain identical to those described below.
} 


\subsection{Agent's problem}

Let $s^{t}=\left\{s_{b}, s_{b+1}, \ldots, s_{t}\right\}$ denote the individual history of the shocks for an agent from birth year $b$ up to date $t$, where

$$
s_{j}=\left\{\begin{array}{clc}
\left(b, \varphi, \alpha^{0}, \kappa^{0}, \theta_{b}\right) & \in \mathbb{S}_{b}=\mathbb{N} \times \mathbb{R}^{4} & \text { for } j=b \\
\left(\omega_{j}, \eta_{j}, \theta_{j}\right) & \in \mathbb{S}=\mathbb{R}^{3} & \text { for } j>b
\end{array}\right.
$$

with $s^{t} \in \mathbb{S}_{b} \times \mathbb{S}^{t-b}$.

Let $Q_{t}\left(S ; s^{t}\right)$ denote the price of insurance claims purchased at date $t$ from local (withinisland) insurers by an agent with history $s^{t}$ that deliver one unit of consumption at $t+1$ if and only $s_{t+1} \in S \subseteq \mathbb{S}$. Let $B_{t}\left(s_{t+1} ; s^{t}\right)$ denote the quantity of the claim purchased that pays in individual state $s_{t+1}$. Recall that insurers can also offer contracts indexed to $\left(\eta_{t+1}, \theta_{t+1}\right)$ to agents in other islands. Define $z_{t+1} \equiv\left(\eta_{t+1}, \theta_{t+1}\right)$ where $z_{t+1} \in Z \subseteq \mathbb{Z}=\mathbb{R}^{2}$. Let $Q_{t}^{*}\left(Z ; s^{t}\right)$ denote the price of insurance claims purchased at date $t$ from outside (betweenisland) insurers by an agent with history $s^{t}$ that deliver one unit of consumption at $t+1$ if and only $z_{t+1} \in Z$. Let $B_{t}^{*}\left(z_{t+1} ; s^{t}\right)$ denote the quantity of the claim purchased from outside insurers that pays upon the realization $z_{t+1}$. The agent's budget constraint is given by

$$
\begin{aligned}
\lambda\left[w_{t}\left(s^{t}\right) h_{t}\left(s^{t}\right)\right]^{1-\tau}+d_{t}\left(s^{t}\right)= & c_{t}\left(s^{t}\right)+\int_{\mathbb{S}} Q_{t}\left(s_{t+1} ; s^{t}\right) B_{t}\left(s_{t+1} ; s^{t}\right) d s_{t+1} \\
& +\int_{\mathbb{Z}} Q_{t}^{*}\left(z_{t+1} ; s^{t}\right) B_{t}^{*}\left(z_{t+1} ; s^{t}\right) d z_{t+1},
\end{aligned}
$$

where realized wealth at node $s^{t}=\left(s^{t-1}, s_{t}\right)$ is given by

$$
d_{t}\left(s^{t}\right)=\delta^{-1}\left[B_{t-1}\left(s_{t} ; s^{t-1}\right)+B_{t-1}^{*}\left(\eta_{t}, \theta_{t} ; s^{t-1}\right)\right] .
$$

The problem for an agent entering the labor market at date $b$ is to maximize (1) subject to a sequence of budget constraints of the form (6), and the wage process. In addition, agents face limits on borrowing that rule out Ponzi schemes, and non-negativity constraints on consumption and hours worked.

\subsection{Competitive equilibrium}

Given sequences $\left\{F_{\varphi t}, F_{\alpha^{0} t}, F_{\kappa^{0} t} ; F_{\omega t}, F_{\eta t}, F_{\theta t}\right\}$, a competitive equilibrium is a set of allocations $\left\{c_{t}\left(s^{t}\right), h_{t}\left(s^{t}\right), d_{t}\left(s^{t}\right), B_{t}\left(\cdot ; s^{t}\right), B_{t}^{*}\left(\cdot ; s^{t}\right)\right\}$ and prices $\left\{Q_{t}\left(S ; s^{t}\right), Q_{t}^{*}\left(Z ; s^{t}\right)\right\}$ for all dates $t$, all histories $s^{t} \in \mathbb{S}_{b} \times \mathbb{S}^{t-b}$, and all $S \subseteq \mathbb{S}, Z \subseteq \mathbb{Z}$ such that (i) allocations maximize expected lifetime utility, (ii) insurance markets clear, and (iii) the economy-wide markets for the final good and labor services clear. 
Proposition 1 [COMPETITIVE EQUILIBRIUM] There exists a competitive equilibrium characterized as follows:

(i) There is no insurance trade between islands: $B_{t}^{*}\left(Z ; s^{t}\right)=0$ for all $Z$ and all $s^{t}$.

(ii) Consumption and hours are given by

$$
\begin{aligned}
\log c_{t}\left(s^{t}\right) & =-(1-\tau) \widehat{\varphi}+(1-\tau)\left(\frac{1+\widehat{\sigma}}{\widehat{\sigma}+\gamma}\right) \alpha_{t}+\mathcal{C}_{t}^{a} \\
\log h_{t}\left(s^{t}\right) & =-\widehat{\varphi}+\left(\frac{1-\gamma}{\widehat{\sigma}+\gamma}\right) \alpha_{t}+\frac{1}{\widehat{\sigma}} \varepsilon_{t}+\mathcal{H}_{t}^{a}
\end{aligned}
$$

where $a=t-b$ is the age of the individual, and $\mathcal{C}_{t}^{a}$ and $\mathcal{H}_{t}^{a}$ are age and date-specific constants (see Appendix A.1), $1 / \widehat{\sigma} \equiv(1-\tau) /(\sigma+\tau)$ is a tax-modified Frisch elasticity, and $\widehat{\varphi} \equiv \varphi /(\sigma+\gamma+\tau(1-\gamma))$ is a rescaled preference weight.

(iii) The prices of insurance claims are given by

$$
\begin{aligned}
Q_{t}\left(S ; s^{t}\right) & =Q_{t}(S)=\beta \exp \left(-\gamma \Delta \mathcal{C}_{t+1}\right) \int_{S} \exp \left(-\gamma(1-\tau) \frac{1+\widehat{\sigma}}{\widehat{\sigma}+\gamma} \omega_{t+1}\right) d F_{s, t+1}(9) \\
Q_{t}^{*}\left(Z ; s^{t}\right) & =Q_{t}^{*}(Z)=\operatorname{Pr}\left(\left(\eta_{t+1}, \theta_{t+1}\right) \in Z\right) \times Q_{t}(\mathbb{S}),
\end{aligned}
$$

where $F_{\text {st }}$ is the joint distribution over $(\omega, \eta, \theta)$ at date $t, Q_{t}(\mathbb{S})$ is the price of a risk-free bond, and $\Delta \mathcal{C}_{t+1} \equiv \mathcal{C}_{t+1}^{a+1}-\mathcal{C}_{t}^{a}$ is independent of age.

\section{Proof. See Appendix A.1.}

Part (i) of Proposition 1 says that there is an equilibrium in which all trade takes place within islands. This result implies zero private insurance against the $\alpha_{t}$ component of idiosyncratic wage risk, because shocks to $\alpha_{t}$ are common to all members of an island. Note, in particular, that there is no self-insurance (via a non-contingent bond traded across islands) against these shocks. Because of perfect insurance against shocks to $\varepsilon_{t}$, in this equilibrium there is a sharp dichotomy between one type of risk against which there is no private insurance, and another that is fully privately insured. In what follows, we will use the term (privately) "uninsurable" to denote the $\omega$ shock and the initial draws $\alpha^{0}$ and $\varphi$, and the term "insurable" to denote the $(\eta, \theta)$ shocks and the initial draw $\kappa^{0}$. When the variance of insurable shocks is zero, equilibrium allocations correspond to autarky. When the variance of uninsurable shocks is zero, there is complete insurance against idiosyncratic risk. In the general case, when both types of shocks have positive variance, private insurance is partial. 
Part (ii) characterizes equilibrium allocations for consumption and hours worked in closed form. These expressions indicate that the vector of cumulated values for the shocks $\left(\alpha_{t}, \varepsilon_{t}\right)$ together with $\varphi$ and age $a$ contain sufficient information to fully describe an individual's equilibrium choices at node $s^{t}$. The power of this result lies in the fact that these are all exogenous states. Crucially, individual wealth is a redundant state variable, in the sense that it is also only a function of $\left(a, \varphi, \alpha_{t}, \kappa_{t}, \theta_{t}\right)$. The expression for wealth $d_{t}$ is in Appendix A.1. Note that no distributional assumptions for wage shocks or preference heterogeneity are required to deliver these functional forms for equilibrium allocations. ${ }^{9}$

Part (iii) describes the insurance prices supporting this equilibrium. The key result is that the prices of insurance contracts on the inter-island market are actuarially fair, in the sense that they are equal to event-specific probabilities times the risk-free bond price $Q_{t}(\mathbb{S})$ - the price at which all agents are indifferent between borrowing and lending on the margin. At these prices, agents have no incentive to buy insurance from or sell insurance to agents on other islands, thereby supporting the no-trade result in part (i).

The logic of the proof for Proposition 1 is as follows. We first guess that all insurance claims are traded within island and that there is no insurance trade between islands. Hence aggregate island-level net savings is zero on each island. Because insurance markets are complete within an island, we then solve for the island-specific allocations via a simple static equal-weight planner's problem. ${ }^{10}$ We can use planner problems to solve for withinisland allocations, notwithstanding the presence of progressive distortionary taxation at the economy-wide level, because each island planner controls a measure zero of aggregate resources and therefore takes the tax/transfer function as exogenous. With expressions for consumption and hours worked in hand, we use the agent's intertemporal first-order condition to compute the implied (potentially island-specific) insurance prices. Finally, we verify that agents on every island assign the same value to any insurance contract that can be traded, and thus that there are no gains from inter-island trade.

\footnotetext{
${ }^{9}$ The distributions only affect the separable constants $\mathcal{C}_{t}^{a}$ and $\mathcal{H}_{t}^{a}$. We implicitly assume that the distributions imply finite values for these constants. The absence of an explicit solution for $\mathcal{C}_{t}^{a}$ and $\mathcal{H}_{t}^{a}$ is no obstacle for the empirical analysis, since the constants can be modeled through age and time dummies in individual consumption and hours observations.

${ }^{10}$ Within-island allocations can be determined using equal-weight island-level planning problems because we defined an island as a group of agents with the same birth date $b$, common initial conditions $\left(\varphi, \alpha^{0}, \kappa^{0}\right)$, and a common sequence $\left\{\omega_{s}\right\}_{s=b+1}^{\infty}$. As a result, groups are fixed over time. However, all of our theoretical results apply under less restrictive assumptions on the definition of an island. In particular, the only fundamental requirement is that all members of an island in period $t$ experience the same realization of $\omega_{t+1}$, but not necessarily the same sequence thereafter. Thus island membership need not be fixed over time.
} 
Interpreting consumption and hours allocations The impact of the preference parameter $\varphi$ on hours and consumption is readily interpreted: a stronger relative distaste for work (higher $\varphi$ ) reduces labor supply, which transmits to earnings and consumption. We now turn to the impact of wage shocks on the allocations for hours and consumption.

Hours worked are increasing in the insurable component $\varepsilon_{t}=\kappa_{t}+\theta_{t}$, and the response of hours to shocks to $\varepsilon_{t}$ is defined by the tax-modified Frisch elasticity, $1 / \widehat{\sigma} \equiv(1-\tau) /(\sigma+\tau)$. Progressive taxation $(\tau>0)$ lowers the tax-modified Frisch elasticity because it reduces the return to increasing hours worked in response to a rise in pre-tax wages. While full insurance with respect to $\varepsilon_{t}$ rules out any income effect on hours worked, uninsurable permanent shocks to $\alpha_{t}$ do have an income effect which is regulated by $\gamma$. If $\gamma>1$, the income effect dominates the substitution effect, and hours worked decline in response to an increase in $\alpha_{t}$. If $\gamma<1$, the substitution effect dominates and hours increase.

Individual consumption is independent of $\varepsilon_{t}$, since these shocks are fully insured. The response of consumption to uninsurable wage shocks depends on the response of hours worked and the progressivity of taxation. Stronger income effects (larger $\gamma$ ) reduce the pass-through from wage shocks to consumption, as does more progressive taxation (larger $\tau$ ). The expression for individual consumption is not what the permanent income hypothesis would imply. Consumption is still a random walk, but some permanent shocks (innovations $\eta_{t}$ ) are insured and thus do not affect consumption. In other words, consumption in our model exhibits "excess smoothness" (as originally defined by Campbell and Deaton, 1989). It is precisely this feature of the data that has motivated a large amount of recent research aimed at developing "partial insurance" models that lie in between the bond economy and complete markets (e.g., Krueger and Perri, 2006; Attanasio and Pavoni, 2011; Ales and Maziero, 2009).

Alternative decentralizations Our characterization of equilibrium assumes that all within-island risk sharing arises from explicit markets and state-contingent financial income flows. However, one could support the same allocations for consumption and hours worked through non-market insurance mechanisms (e.g., within-family state-contingent transfers). Similarly, if agents could perfectly foresee future innovations $\left(\eta_{t}, \theta_{t}\right)$, then a non-contingent bond traded within islands would suffice to allow them to perfectly smooth consumption in response to these wage changes. We use the label "insurable shocks" as a catchall for both 
insurable and forecastable wage changes. ${ }^{11}$

\subsection{Tractability of the framework}

With few exceptions, incomplete markets models do not admit an analytical solution and numerical methods are required to solve for equilibrium allocations. ${ }^{12}$ In this section we explain how we retain tractability, and we relate this result to the existing literature. Readers who are more interested in the empirical application can skip directly to Section 3.

\subsubsection{How we retain tractability}

There are two keys to tractability in our framework: (i) individual wealth is a redundant state variable, and (ii) agents have access to perfect private insurance against some shocks and no private insurance against others.

Why wealth is a redundant state The reason individual wealth is a redundant state variable is twofold. First, even though the within-island equilibrium wealth distribution is non-degenerate, allocations can be characterized without reference to it: full insurance within the island implies that within-island allocations can be derived by solving an islandlevel planner problem with an equal-weight welfare function corresponding to common initial asset positions for all agents, subject to an island-level resource constraint.

Second, the inter-island wealth distribution does not show up in allocations because, in equilibrium, this distribution remains degenerate at zero. This second argument can be explained in three simple steps. To understand why there is no asset trade between islands, it is sufficient to understand why there is no trade in a risk-free bond. ${ }^{13}$ Let $r_{t+1}=-\log Q_{t}(\mathbb{S})$

\footnotetext{
${ }^{11}$ Cunha, Heckman, and Navarro (2005) and Guvenen and Smith (2010), among others, explain the difficulty in distinguishing between insurable and predictable shocks.

${ }^{12}$ In standard (intractable) incomplete markets models, decision rules depend on wealth, and the distribution of wealth is endogenous and must be solved for numerically. The literature has followed three alternative routes to avoid this outcome. The first is to assume a statistical model for income risk (permanent, multiplicative shocks) such that the equilibrium wealth distribution remains degenerate at zero (Constantinides and Duffie, 1996). The second is to assume a preference specification - quadratic or in the constant absolute risk aversion (CARA) class - such that the precautionary motive for saving is either zero or independent of wealth (Caballero, 1990). The third is to allow agents to control the amount of idiosyncratic risk that they face such that equilibrium exposure to idiosyncratic risk is proportional to wealth, given CRRA preferences (Krebs, 2003; Angeletos, 2007). Krebs (2003) allows for human capital accumulation, so that agents can control the composition between (safe) physical and (risky) human wealth independently of total wealth by making savings choices in both assets. Angeletos (2007) models idiosyncratic risk to entrepreneurial business income rather than labor income. In his model agents control portfolio exposure to idiosyncratic risk by adjusting the quantity of entrepreneurial capital in total savings.

${ }^{13}$ Recall that inter-island insurance prices are simply event-specific probabilities times the bond price.
} 
denote the equilibrium interest rate and $\rho=-\log \beta$ the discount rate. In the model, individuals have three saving motives: an intertemporal motive proportional to the gap between $r_{t+1}$ and $\rho$, a smoothing motive linked to expected earnings growth over the life cycle, and a precautionary motive that is a function of the variance of uninsurable islandlevel shocks $v_{\omega, t+1}$. Importantly, each of these three factors applies with the same force on all islands. The strength of the intertemporal motive is given by the term $\left(r_{t+1}-\rho\right) / \gamma$, common across agents. All islands have the same smoothing motive, because island-level expected earnings growth is independent of age and of the current wage. The precautionary motive is the same because all agents face the same variance for the uninsurable component of wages. Consequently, there exists an economy-wide interest rate $r_{t+1}$ at which, in equilibrium, the (negative) intertemporal motive exactly offsets the (negative) smoothing motive and the (positive) precautionary motive, and no agent wants to either borrow or lend across islands.

To gain more intuition, it is useful to make a specific distributional assumption. If each variable $x_{t} \in\left(\omega_{t}, \eta_{t}, \theta_{t}\right)$ is distributed Normally, $x_{t} \sim N\left(-v_{x t} / 2, v_{x t}\right)$, then asset prices can be derived in closed form. Focusing, for simplicity, on the special case $\tau=0$, we have

$$
r_{t+1}=\rho-\gamma \frac{1+\sigma}{\sigma+\gamma}\left[\left(\gamma \frac{1+\sigma}{\sigma+\gamma}+1\right) \frac{v_{\omega, t+1}}{2}-\frac{\Delta \operatorname{var}_{t+1}(\varepsilon)}{2 \sigma}\right],
$$

where $\Delta \operatorname{var}_{t+1}(\varepsilon) \equiv v_{\eta, t+1}+v_{\theta, t+1}-v_{\theta t}$ is the increase in the variance of the insurable component of the wage for all cohorts between years $t$ and $t+1$.

Let us begin by assuming that $\sigma \rightarrow \infty$ (inelastic labor supply). In the absence of islandlevel risk $\left(v_{\omega, t+1}=0\right)$, the equilibrium interest rate would satisfy the standard condition of a complete-market endowment economy, $r_{t+1}=\rho$. In the presence of island-level risk, expression (10) simplifies to $\left(r_{t+1}-\rho\right) / \gamma+v_{\omega, t+1}(1+\gamma) / 2=0$. The first term measures the intertemporal motive to dis-save, since the rate of time preference exceeds the equilibrium interest rate. The second term, capturing the precautionary motive for saving, is equal to half the variance of the island-level productivity shocks times the coefficient of relative prudence, $(1+\gamma)$. The equilibrium interest rate is such that the two saving motives exactly offset. When $\sigma$ is finite, its effect on $r_{t+1}$ depends on the value for $\gamma$. If $\gamma>1$, then hours respond negatively to uninsurable shocks (see equation 8). In this case, a higher Frisch elasticity reduces the precautionary saving motive, since labor supply provides a useful hedge against risk. $^{14}$

\footnotetext{
${ }^{14}$ Progressivity, $\tau>0$, reduces the private precautionary saving motive (see eq. (A5) in the Technical Appendix A).
} 
Insurance dichotomy and island structure We now explain why and when the island structure is required to construct an equilibrium in which some shocks are perfectly insured while others remain uninsured.

Recall that the uninsurable and insurable components $\alpha_{t}$ and $\varepsilon_{t}$ enter the level wage multiplicatively. This means that to perfectly insure $\varepsilon_{t}$ shocks, individuals must be able to scale their purchases of insurance against these shocks to their future realizations for uninsurable shocks. If $\varepsilon_{t}$ were i.i.d., so that insurable shocks lasted only one period, it would be necessary to index insurance purchases only to the one-period-ahead innovation $\omega_{t+1}$. This could be achieved by simply assuming that agents first observe the innovation $\omega_{t+1}$, and then trade - economy-wide - insurance claims contingent on the realization of the transitory component $\theta_{t+1}$. Thus in that case it is not necessary to partition the economy into islands. However, in our model $\varepsilon_{t}$ has a unit-root component, and an innovation $\eta_{t+1}$ has a permanent effect on future earnings. This requires linking insurance payouts to the agent's future sequence of uninsurable shocks. The island structure is a technical expedient allowing agents to trade an unrestricted set of insurance claims, thereby enabling perfect insurance against the $\varepsilon_{t}$ component. At the same time, because unrestricted contracts are only exchanged within the island, the structure prevents agents from pooling the island-level risk. ${ }^{15}$

\subsubsection{Relation to Constantinides and Duffie (1996)}

Constantinides and Duffie (1996), henceforth CD, is an important forebear of our model. The key insight of CD is that a no-trade equilibrium exists when: (1) the exogenous process for disposable income is a multiplicative unit root with innovations drawn from a distribution common to all agents, (2) preferences are in the power utility class, (3) assets in zero net supply, and agents are endowed with zero initial wealth. ${ }^{16}$ We extend CD's environment in four dimensions that are important for a quantitative study of risk sharing.

First, our primitive exogenous stochastic process is over hourly wages and also includes a

\footnotetext{
${ }^{15}$ In our competitive equilibrium, transfers associated with permanent insurable shocks are linked to the entire future sequence of uninsurable shocks, even though the decentralization is achieved with one-period within-island insurance contracts. This is a parallel to the standard result that a sequential equilibrium with one-period Arrow securities gives rise to the same allocation as the Arrow-Debreu equilibrium with trade only at date zero.

${ }^{16}$ Both CD's model and ours can have assets in positive net supply in a trivial case, namely when agents are endowed at birth with a unit of the market portfolio and pay a lump-sum tax each period equal to the dividend on the market portfolio each period. In equilibrium, agents never trade away from their initial holding of the market portfolio, rendering the allocations (7)-(8) unchanged.
} 
transitory component beyond the unit root. Gross earnings are endogenous since individuals control their labor supply. Showing that the no-trade result extends to preferences defined over labor supply as well as consumption is important because, as will become clear shortly, data on hours worked are a rich source of information on the nature of risk and risk sharing. In Heathcote, Storesletten, and Violante (2011b) we generalize the preference class under which the no-trade result holds beyond our baseline specification (2). We provide a simple static sufficient condition that can be used to check whether there exists an equilibrium with no inter-island trade, for any particular utility function defined over consumption and hours worked. We use this condition to show that the no-trade result extends to GreenwoodHercowitz-Huffman, Cobb-Douglas, and recursive Epstein-Zin preferences. These alternative specifications also deliver closed-form expressions for equilibrium allocations.

Second, we allow for progressive taxation, which allows us to quantify how much insurance takes place through the government and how much is provided privately.

Third, agents in our model differ with respect to preferences, in addition to productivity. This feature is important because we do not want to impose a priori that the entire crosssectional dispersion in consumption and hours worked is driven by dispersion in wages.

Finally, and most importantly, in our economy some risks are privately insurable within islands, so our version of the no-trade result applies across groups rather than across individuals. Hence, our model allows for partial consumption insurance against disposable earnings shocks - a critical requirement for bringing the model to the data successfully (as shown by Blundell, Preston, and Pistaferri, 2008). In contrast, the most direct interpretation of the CD model is that theirs is a world with no risk sharing in which each individual consumes his or her endowment. An alternative interpretation is that their postulated endowment process is "post-trade" and incorporates non-modeled insurance against fundamental shocks. Relative to this alternative interpretation, the advantage of our setup is that we explicitly model and quantify the channels of insurance available to households: labor supply (from wages to earnings), progressive taxation (from earnings to disposable income), and private insurance (from disposable income to consumption).

\section{Cross-sectional implications}

The model has thus far abstracted from variation in household composition, while actual households in the data vary with respect to household size and the number of potential 
workers. Moreover, measurement error is pervasive in micro data. In this section, we first describe how to "augment" our theoretical allocations to address these two issues. Next, we use these augmented theoretical allocations to derive, and interpret, closed-form expressions for (co-)variances of the equilibrium cross-sectional joint distribution of consumption, hours, and wages - the key moments used for model identification and estimation.

\subsection{Augmented theoretical allocations}

Modeling household composition To address the first issue, we generalize the model to explicitly incorporate variation in household size. This extension delivers a theoretically coherent approach for controlling for household composition in the data.

Let $g$ and $k$ denote the number of adults (grown-ups) and children (kids) in a particular household. All members of a given household reside on the same island. Let $e(g, k)$ be a function that defines the economies of scale enjoyed by a household of type $(g, k)$ such that effective per-person consumption is given by household consumption $c$ divided by $e(g, k)$, where $e(1,0)$ is normalized to unity. Children receive no weight in household utility. Thus period utility for a household of type $(\varphi, g, k)$ is given by

$$
u\left(c,\left\{h_{i}\right\}_{i=1}^{g} ; \varphi, g, k\right)=\frac{g}{1-\gamma}\left(\frac{c}{e(g, k)}\right)^{1-\gamma}-\frac{\exp (\varphi)}{1+\sigma} \sum_{i=1}^{g} h_{i}^{1+\sigma} .
$$

One could make alternative assumptions regarding whether agents can insure ex ante against the type $(g, k)$ of household to which they are allocated. In Appendix A.2, we solve for allocations in the two polar cases where there is full insurance and no insurance against $(g, k)$, respectively. The key difference between the two models is that the full insurance model implies that hours worked should be independent of household composition, while the no-insurance model implies that hours should vary systematically with household size (assuming $\gamma \neq 1$ ). The reason household type does not affect equilibrium hours in the insurable household composition model is that household type has no impact on productivity or the disutility of labor effort, and thus it would be inefficient for individuals in different-size households to work different numbers of hours.

Motivated by this distinction, we experimented with regressing log hours on household composition dummies. Conditional on hours being positive, household composition explains essentially none of the observed variation in hours worked on the intensive margin, which is evidence in favor of the insurable model of household composition. ${ }^{17}$

\footnotetext{
${ }^{17}$ It is possible that household composition is more important in accounting for participation decisions on
} 
In Appendix A.2 we show that with full insurance against household composition, total consumption is given by

$$
\log c_{t}^{a}\left(s^{t} ; g, k\right)=\log c_{t}^{a}\left(s^{t} ; 1,0\right)+D(g, k)
$$

where $\log c_{t}^{a}\left(s^{t} ; 1,0\right)$, consumption for a single-adult household, is given by equation $(7)$, and $D(g, k)$ is given by

$$
D(g, k) \equiv \frac{1}{\gamma} \log g-\left(\frac{1-\gamma}{\gamma}\right) \log e(g, k) .
$$

From this expression it is clear that if $\gamma=1$ or $e(g, k)=g$, then households are allocated consumption exactly in proportion to the number of adults $g$, so there are no transfers between households of different size. Suppose there are economies of scale from additional adults (so that $e(g, 0)<g$ for $g>1$ ). Then larger households are allocated less consumption per adult than smaller households if and only if $\gamma>1$. On the one hand, economies of scale make it inexpensive to increase effective consumption $c / e(g, k)$ for large households in the limit $\gamma \rightarrow 0$ this effect makes it efficient to allocate all consumption to the largest households. On the other hand, for $\gamma>0$, economies of scale mean that for the same level of consumption per adult, larger households enjoy a lower marginal utility of consumption. If $\gamma>1$ this second effect dominates.

With prior knowledge of the appropriate equivalence scale $e(g, k)$ and the risk aversion parameter $\gamma$, one could purge variation in household size from the data by applying eq. (12) directly. Instead we choose to be agnostic ex ante about the function $e(g, k)$ and simply regress log household consumption on a full set of composition dummies. We do not regress hours (or wages) on household composition dummies because they do not appear in the corresponding theoretical expressions, given insurance against household size. In the same consumption regression, we also strip out the age/time dummies $\mathcal{C}_{t}^{a}$ (by including a quartic polynomial in age and a full set of year dummies), and run similar regressions (minus the composition dummies) for individual wages and hours. ${ }^{18}$

Measurement error We assume that consumption, earnings, and hours worked are measured with error and that this error is classical, i.e., i.i.d. over time and across agents. The $\log$ of the observed value for variable $x_{t}$ is then $\log \hat{x}_{t}=\log x_{t}+\mu_{t}^{x}$, where measurement

the extensive margin. In Section 6 we describe how to extend the model to allow for a participation decision without sacrificing tractability.

${ }^{18}$ Note that the polynomial in age also eliminates life-cycle effects in wages, hours, and consumption that we do not model. 
error $\mu_{t}^{x}$ has mean zero and variance $v_{\mu x}$. While we directly observe consumption, hours, and earnings, we compute hourly wages as earnings divided by hours. Hence measurement error in hourly wages reflects errors in both earnings and hours.

Augmented allocations Augmented log allocations at time $t$ are therefore given by

$$
\begin{aligned}
\log \hat{w}_{t} & =\alpha_{t}+\kappa_{t}+\theta_{t}+\mu_{t}^{y}-\mu_{t}^{h} \\
\log \hat{c}_{t} & =-(1-\tau) \widehat{\varphi}+(1-\tau)\left(\frac{1+\widehat{\sigma}}{\widehat{\sigma}+\gamma}\right) \alpha_{t}+\mu_{t}^{c} \\
\log \hat{h}_{t} & =-\widehat{\varphi}+\left(\frac{1-\gamma}{\widehat{\sigma}+\gamma}\right) \alpha_{t}+\frac{1}{\widehat{\sigma}} \varepsilon_{t}+\mu_{t}^{h},
\end{aligned}
$$

where, recall, $\widehat{\varphi}$ denotes the rescaled preference weight.

\subsection{Interpreting cross-sectional variances and covariances}

With these allocations in hand, we can express in closed form cross-sectional moments of the joint equilibrium distribution of wages, hours, and consumption. These theoretical moments represent an attractive feature of our framework, since they allow us to transparently interpret the dynamics of their empirical counterparts over the life cycle and over time.

We will focus on variances and covariances across all agents of age $a$ at date $t$. These moments reflect dispersion both within and between islands. An important theoretical property of our framework (see Section 4.1) is that the information contained in the aggregate crosssectional (co-)variances of wages, hours, and consumption is sufficient to identify all model parameters and to quantify risk sharing. Thus we do not need to determine the empirical counterparts to model islands - a daunting task, since the theory puts very few restrictions on the notion of an island: it is just a group of agents pooling a subset of idiosyncratic shocks at a point in time.

We start from the moments in levels, which we call the "macro moments" and then move to those in differences, which we will refer to as the "micro moments."

Macro moments Let $\operatorname{var}_{t}^{a}(\alpha)$ denote the within-cohort variance of cumulated permanent uninsurable shocks (up until) period $t$ for agents of age $a$ :

$$
\operatorname{var}_{t}^{a}(\alpha)=v_{\alpha^{0}, t-a}+\sum_{j=0}^{a-1} v_{\omega, t-j}
$$

Similarly, let $\operatorname{var}_{t}^{a}(\widehat{\varphi})=v_{\widehat{\varphi}, t-a}$ denote the cohort $(t-a)$-specific variance of the rescaled 
preference weights, and let $\operatorname{var}_{t}^{a}(\varepsilon)=v_{\kappa^{0}, t-a}+\sum_{j=0}^{a-1} v_{\eta, t-j}+v_{\theta t}$ be the variance of the insurable component of the wage for cohorts of age $a$ in year $t$.

The macro moments for wages and hours for age group $a$ at date $t$ are, respectively,

$$
\begin{aligned}
\operatorname{var}_{t}^{a}(\log \hat{w}) & =\operatorname{var}_{t}^{a}(\alpha)+\operatorname{var}_{t}^{a}(\varepsilon)+v_{\mu y}+v_{\mu h} \\
\operatorname{var}_{t}^{a}(\log \hat{h}) & =\operatorname{var}_{t}^{a}(\widehat{\varphi})+\left(\frac{1-\gamma}{\widehat{\sigma}+\gamma}\right)^{2} \operatorname{var}_{t}^{a}(\alpha)+\frac{1}{\widehat{\sigma}^{2}} \operatorname{var}_{t}^{a}(\varepsilon)+v_{\mu h} \\
\operatorname{cov}_{t}^{a}(\log \hat{w}, \log \hat{h}) & =\left(\frac{1-\gamma}{\widehat{\sigma}+\gamma}\right) \operatorname{var}_{t}^{a}(\alpha)+\frac{1}{\widehat{\sigma}} \operatorname{var}_{t}^{a}(\varepsilon)-v_{\mu h} .
\end{aligned}
$$

The variance of measured wages is the sum of variances of the orthogonal productivity components, plus the variances of measurement error in earnings and hours. The variance of hours has four components. First, the more heterogeneity in the taste for leisure $\varphi$, the larger is the cross-sectional dispersion in hours. Second, the variance of the uninsurable shock translates into hours dispersion proportionately to $1-\gamma$. As $\gamma \rightarrow 1$ (the log-consumption case), uninsurable shocks have no effect on hours. Third, the variance of the insurable shocks increases hours dispersion in proportion to the (squared) tax-modified Frisch elasticity. Finally, measurement error in hours contributes positively to observed dispersion.

The covariance between wages and hours has three components. The effect of uninsurable wage shocks on this covariance depends on the value for $\gamma$. If $\gamma>1$, then uninsurable shocks decrease the wage-hours covariance, since strong income effects induce low wage (uninsured) workers to work longer hours. Insurable shocks, by contrast, make hours and wages move together. Measurement error in hours reduces the observed covariance between hours and wages (earnings divided by hours).

We now turn to the moments involving consumption:

$$
\begin{aligned}
\operatorname{var}_{t}^{a}(\log \hat{c}) & =(1-\tau)^{2} \operatorname{var}_{t}^{a}(\widehat{\varphi})+(1-\tau)^{2}\left(\frac{1+\widehat{\sigma}}{\widehat{\sigma}+\gamma}\right)^{2} \operatorname{var}_{t}^{a}(\alpha)+v_{\mu c} \\
\operatorname{cov}_{t}^{a}(\log \hat{h}, \log \hat{c}) & =(1-\tau) \operatorname{var}_{t}^{a}(\widehat{\varphi})+\frac{(1-\tau)(1+\widehat{\sigma})(1-\gamma)}{(\widehat{\sigma}+\gamma)^{2}} \operatorname{var}_{t}^{a}(\alpha) \\
\operatorname{cov}_{t}^{a}(\log \hat{w}, \log \hat{c}) & =(1-\tau)\left(\frac{1+\widehat{\sigma}}{\widehat{\sigma}+\gamma}\right) \operatorname{var}_{t}^{a}(\alpha) .
\end{aligned}
$$

The variance of consumption is increasing in the variance of uninsurable preference heterogeneity and uninsurable wage shocks, as expected. Progressive taxation $(\tau>0)$ reduces the variance of consumption for a given $\operatorname{var}_{t}^{a}(\alpha)$. The role of labor supply depends on the value for $\gamma$ : for $\gamma>1$ a lower $\sigma$ (higher Frisch) reduces consumption dispersion because labor supply offsets uninsurable wage shocks and dampens their impact on earnings. 
The covariance between hours and consumption is increasing in the degree of preference heterogeneity, since individuals with higher $\varphi$ work relatively few hours and thus earn and consume relatively less. The effect of uninsurable wage risk depends on the value of $\gamma$ : when $\gamma>1$, a positive uninsurable shock reduces hours worked but increases consumption.

The covariance between consumption and wages depends only on uninsurable wage shocks: fluctuations in uninsurable productivity affect both wages and consumption in the same direction. As expected, progressive taxation reduces this covariance. ${ }^{19}$

Dispersion over the life cycle Let $\Delta v a r_{t}^{a}(\log \hat{x})=v a r_{t}^{a}(\log \hat{x})-v a r_{t-1}^{a-1}(\log \hat{x})$ be the within-cohort change (i.e., between age $a-1$ in year $t-1$ and age $a$ in year $t$ ) in the variance of $\log \hat{x}$. The model has sharp predictions for the life-cycle evolution of dispersion:

$$
\begin{aligned}
\Delta \operatorname{var}_{t}^{a}(\log \hat{w}) & =v_{\omega t}+v_{\eta t}+\Delta v_{\theta t} \\
\Delta \operatorname{var}_{t}^{a}(\log \hat{h}) & =\left(\frac{1-\gamma}{\widehat{\sigma}+\gamma}\right)^{2} v_{\omega t}+\frac{1}{\widehat{\sigma}^{2}}\left(v_{\eta t}+\Delta v_{\theta t}\right) \\
\Delta \operatorname{cov}_{t}^{a}(\log \hat{w}, \log \hat{h}) & =\left(\frac{1-\gamma}{\widehat{\sigma}+\gamma}\right) v_{\omega t}+\frac{1}{\widehat{\sigma}}\left(v_{\eta t}+\Delta v_{\theta t}\right) \\
\Delta \operatorname{var}_{t}^{a}(\log \hat{c}) & =(1-\tau)^{2}\left(\frac{1+\widehat{\sigma}}{\widehat{\sigma}+\gamma}\right)^{2} v_{\omega t} \\
\Delta \operatorname{cov}_{t}^{a}(\log \hat{h}, \log \hat{c}) & =(1-\tau) \frac{(1-\gamma)(1+\widehat{\sigma})}{(\widehat{\sigma}+\gamma)^{2}} v_{\omega t} \\
\Delta \operatorname{cov}_{t}^{a}(\log \hat{w}, \log \hat{c}) & =(1-\tau)\left(\frac{1+\widehat{\sigma}}{\widehat{\sigma}+\gamma}\right) v_{\omega t} .
\end{aligned}
$$

None of these moments involve measurement error, reflecting our assumption that the variance of measurement error is independent of age and time. Moreover, because all shocks in our economy are either permanent or i.i.d., all of these moments are independent of age.

The rise in wage inequality over the life cycle is determined by the variance of the innovations to the permanent insurable and uninsurable components, and by the change in the variance of the transitory insurable component. Wage dispersion will increase over the life cycle as permanent shocks cumulate. The model suggests that the variance of hours should be increasing over the life cycle for the same reasons as wages, though with different weights

\footnotetext{
${ }^{19}$ Since we have filtered out differences in mean values for allocations across age groups, the expressions for dispersion in the entire cross section are identical to those above, but without the age $a$ superscripts. This follows from the variance decomposition $\operatorname{var}_{t}(x)=\mathbb{E}\left[\operatorname{var}_{t}^{a}(x)\right]+\operatorname{var}_{t}[\mathbb{E}(x \mid a)]$, where the second term is zero if we abstract from the terms $\mathcal{C}_{t}^{a}$ and $\mathcal{H}_{t}^{a}$ in the allocations. Thus, for example, $\operatorname{var}_{t}(\log \hat{w})=$ $\operatorname{var}_{t}(\alpha)+\operatorname{var}_{t}(\varepsilon)+v_{\mu y}+v_{\mu h}$, where $\operatorname{var}_{t}(\alpha)=(1-\delta) \sum_{a=0}^{\infty} \delta^{a} \operatorname{var}_{t}^{a}(\alpha)$ is the unconditional cross-sectional variance of the uninsurable component of $\log$ wages, and $\operatorname{var}_{t}(\varepsilon)$ is the corresponding variance for the insurable component of wages.
} 
on the insurable and uninsurable permanent variances. In the log-consumption utility case $(\gamma=1)$, only the former matters for hours.

Whether the covariance between wages and hours rises or falls over the life cycle depends on risk aversion and the relative size of permanent and transitory innovations. When $\gamma>1$, the cumulation of permanent uninsurable shocks pushes the covariance down as individuals age, while the cumulation of permanent insurable shocks pulls the covariance up.

The change in the variance of consumption over the life cycle is determined by the variance of uninsurable productivity shocks. The uninsurable-wage-shock coefficient for consumption is exactly one when $\tau=0$ and either $\gamma=1$ or $\sigma \rightarrow \infty$.

When $\gamma>1$, hours move up in response to a negative uninsurable wage shock, while consumption moves down, driving the consumption-hours covariance down over the life cycle as $\operatorname{var}_{t}^{a}(\alpha)$ rises with age. Finally, the model predicts that the covariance between consumption and wages will increase over the life cycle, in proportion to $v_{\omega t}$.

Micro moments Micro moments are computed as variances and covariances of individual changes in $\log$ wages and $\log$ hours between $t-1$ and $t .^{20}$ Let $\Delta \log \hat{x}_{t} \equiv \log \hat{x}_{t}-\log \hat{x}_{t-1}$ denote the observed individual growth rate for variable $\hat{x}$, and let $\operatorname{var}_{t}^{a}(\Delta \log \hat{x})$ be its crosssectional variance, for the set of individuals of age $a$ at date $t$ for whom variable $\hat{x}$ is observed at both $t-1$ and $t$ :

$$
\begin{aligned}
\operatorname{var}_{t}^{a}(\Delta \log \hat{w}) & =v_{\omega t}+v_{\eta t}+v_{\theta t}+v_{\theta, t-1}+2 v_{\mu y}+2 v_{\mu h} \\
\operatorname{var}_{t}^{a}(\Delta \log \hat{h}) & =\left(\frac{1-\gamma}{\widehat{\sigma}+\gamma}\right)^{2} v_{\omega t}+\frac{1}{\widehat{\sigma}^{2}}\left(v_{\eta t}+v_{\theta t}+v_{\theta, t-1}\right)+2 v_{\mu h} \\
\operatorname{cov}_{t}^{a}(\Delta \log \hat{w}, \Delta \log \hat{h}) & =\left(\frac{1-\gamma}{\widehat{\sigma}+\gamma}\right) v_{\omega t}+\frac{1}{\widehat{\sigma}}\left(v_{\eta t}+v_{\theta t}+v_{\theta, t-1}\right)-2 v_{\mu h}
\end{aligned}
$$

Again, the model implies that the variances and covariances of individual growth rates should be invariant to age and thus common across cohorts. Similar expressions obtain for second differences in wages and hours. For example, the variance of wage growth over a two-year horizon is

$$
\operatorname{var}_{t}^{a}\left(\Delta^{2} \log \hat{w}\right)=v_{\omega t}+v_{\omega, t-1}+v_{\eta t}+v_{\eta, t-1}+v_{\theta t}+v_{\theta, t-2}+2 v_{\mu y}+2 v_{\mu h}
$$

\footnotetext{
${ }^{20}$ Given the specification of the stochastic process for shocks and measurement error, in the model covariances of the individual changes are all zero beyond lag one. Moreover, we omit moments involving changes in consumption, since we do not use the longitudinal dimension of CEX. The panel aspect of CEX is quite weak. It consists of two, generally noisy, observations spaced nine months apart. See Davis (2003) for a discussion.
} 
As we shall see, such moments are especially useful for exploiting the PSID data in the years when the survey was conducted biannually.

Finally, note that all of our cross-sectional moments are the sum of additively separable terms capturing the roles of preference heterogeneity, insurable productivity shocks, uninsurable productivity shocks, and measurement error. This implies that (co-)variance decompositions are always unique, in sharp contrast to the existing literature (e.g., Keane and Wolpin, 1997; Storesletten, Telmer, and Yaron, 2004a; Heathcote, Storesletten, and Violante 2010b), where decompositions must be obtained by simulation, and where the sequence in which various model ingredients are added or removed typically affects their measured contribution to moments of interest. In Section 4.3 we document our decompositions in detail.

\section{Identification, data, and estimation}

In this section, we first exploit the closed-form cross-sectional moments to prove identification of the model parameters. Next, we describe the data used for the structural estimation, and finally we discuss our estimation method. We estimate all structural parameters except $\delta$ and $\tau$, which are set exogenously. Both macro and micro moments contain valuable information about parameters, and both are used to identify and estimate the model.

\subsection{Identification}

The conditions for identification depend on data availability. We therefore consider an array of different scenarios. Our baseline scenario (Proposition 2 below) is that one has access to an unbalanced panel on wages and hours (e.g., the PSID) and a repeated cross section on wages, hours, and consumption (e.g., the CEX). Next, we consider several variants encompassing alternative data structures.

Proposition 2 [IDENTIFICATION] With an unbalanced panel on wages and hours and a repeated cross section on consumption, wages, and hours from $t=1,, T$, the parame-

ters $\left\{\sigma, \gamma, v_{\mu h}, v_{\mu y}, v_{\mu c}\right\}$ as well as the sequences $\left\{v_{\widehat{\varphi} t}, v_{\alpha^{0} t}\right\}_{t=1}^{T},\left\{v_{\kappa^{0} t}, v_{\theta t}\right\}_{t=1}^{T-1},\left\{v_{\omega t}\right\}_{t=2}^{T}$ and $\left\{v_{\eta t}\right\}_{t=2}^{T-1}$ are identified. The sums $v_{\eta T}+v_{\theta T}$ and $v_{\kappa^{0} T}+v_{\theta T}$ are also identified.

Proof. See Appendix A.3.

We now consider two alternative data structures that reflect additional limitations of available survey data for the United States. The first constraint is that consumption data in 
the CEX are available only from 1980, while the PSID starts in 1967. The second limitation is that, starting in 1996, the PSID becomes biannual. Since we estimate the model by combining the PSID and the CEX, these next two corollaries are important for us.

Corollary 2.1 [LImited CONSUmption DATA] Suppose available data comprise an unbalanced panel on wages and hours from $t=1, \ldots, T$ and a repeated cross section on consumption, wages, and hours for at least two years $\hat{t}$ and $\hat{t}+1$, where $1 \leq \hat{t}<T$. Then, parameter identification is exactly as in Proposition 2.

Corollary 2.2 [BIANnul PANEL DATA] Suppose available data comprise an unbalanced panel on wages and hours and a repeated cross section on wages, hours, and consumption, where the cross-sectional data on consumption are annual for all years $t=1, \ldots, T$, while the panel data on wages and hours are annual only until year $\hat{t}$ and biannual thereafter, i.e., data are available for the years $t=1,2, \ldots, \hat{t}$ and $t=\hat{t}+2, \hat{t}+4, \ldots, T-2, T$. Then, one can identify $\left\{\sigma, \gamma, v_{\mu h}, v_{\mu y}, v_{\mu c}\right\}$, the sequences $\left\{v_{\widehat{\varphi} t}, v_{\alpha^{0} t}\right\}_{t=1}^{T},\left\{v_{\omega t}\right\}_{t=2}^{T},\left\{v_{\theta t}, v_{\kappa^{0} t}\right\}_{t=1}^{\hat{t}},\left\{v_{\eta t}\right\}_{t=2}^{\hat{t}}$, and $\left\{v_{\theta t}, v_{\kappa^{0} t}, v_{\eta, t-1}+v_{\eta t}\right\}$ for the years $t=\hat{t}+2, \hat{t}+4, \ldots, T-2$, as well as the sums $\left\{v_{\eta, T-1}+v_{\eta, T}+v_{\theta, T}\right\}$ and $\left\{v_{\kappa^{0}, T}+v_{\theta, T}\right\}$.

It is also straightforward to prove that, up to the composition of insurable shocks (i.e., the split between $v_{\theta t}, v_{\eta t}$, and $v_{\kappa^{0} t}$ ), the model is also identified with only cross-sectional data on consumption, hours, and wages -for example, with data from the CEX alone. ${ }^{21}$

It is well understood in the literature that consumption data can be used to differentiate between insurable and uninsurable shocks (see, e.g., Attanasio and Davis, 1996; Blundell and Preston, 1998; Guvenen and Smith, 2010). Proposition 2 and its corollaries expand this earlier research by introducing data on hours worked alongside consumption to obtain sharper identification. Why are data on labor supply informative about risk sharing? At a basic level, the logic is that theory has sharply different implications for the response of hours to uninsurable versus insurable shocks, just as for consumption. Moreover, the magnitudes of these responses are mediated by preference parameters. We now prove that, under a weak additional restriction on measurement error, the whole model can be identified without using any consumption data.

\footnotetext{
${ }^{21}$ To see this, note that Step A of the proof of Proposition 2 identifies $\sigma, \gamma,\left\{v_{\omega t}\right\}_{t=2}^{T}$, and $\left\{v_{\eta t}+\Delta v_{\theta t}\right\}_{t=2}^{T}$. Following Step $\mathrm{C}$ of the same proof, one identifies $\left\{v_{\widehat{\varphi} t}, v_{\alpha^{0} t}\right\}_{t=1}^{T}$ and $\left\{v_{\kappa^{0} t}+v_{\theta t}\right\}_{t=1}^{T}$. Measurement error $\left\{v_{\mu y}, v_{\mu h}, v_{\mu c}\right\}$ is identified following Step D.
} 
Proposition 3 [identification With no CONSUmption Data] With an unbalanced panel on wages and hours from $t=1, \ldots, T$, and an external estimate of measurement error in earnings $v_{\mu y}$, all the parameters listed in Proposition 2 are identified.

Proof. See Technical Appendix C.1.

Proposition 3 has two immediate implications. First, with an unbalanced panel, only a very short longitudinal dimension is required: all parameters are identified with a three-year panel. Second, the model could alternatively be estimated with longitudinal data on wages and hours for a single cohort. ${ }^{22}$

We conclude this section by emphasizing that this set of identification results constitutes one of the key payoffs from the tractability of our framework. Typically, identification in estimated structural equilibrium models is discussed only at an informal level, because the mapping from parameters to equilibrium moments can at most be weakly illuminated by numerical experimentation. In contrast, our closed-form expressions for equilibrium allocations deliver explicit analytical links between structural parameters and equilibrium moments, enabling us to prove identification formally and lending transparency to the empirical analysis.

\subsection{Data}

Our data are drawn from two surveys, the Michigan Panel Study of Income Dynamics (PSID), and the Consumer Expenditure Survey (CEX). We use PSID data for interview years 1968-2007 (which refer to calendar years 1967-2006). After the 1997 interview, the PSID becomes biannual, so we only have data for survey years 1968-1997, 1999, 2001, 2003, 2005, and 2007. We use CEX data from the quarterly Interview Surveys. Consistent and continuous data over time are available annually since 1980, hence we restrict attention to the 1980-2006 surveys. $^{23}$

Since we jointly use both PSID and CEX data, we apply the same sample selection criteria to both datasets. Namely, we exclude badly incomplete or highly implausible observations. ${ }^{24}$

\footnotetext{
${ }^{22}$ Therefore, besides the PSID, the model can be estimated on the SIPP or the NLSY. With a two-year panel (for example, the rotating panel of the CPS) all parameters are identified, except for $v_{\eta t}$.

${ }^{23}$ In the PSID, we exclude all PSID oversamples (SEO, Latino) so we do not need sample weights, while for the CEX computations use sample weights throughout.

${ }^{24}$ We drop records if 1 ) there is no information on age for either the head or the spouse, 2) if either the head or spouse has positive labor income but zero annual hours, and 3) if either the head or spouse has an hourly wage less than half of the corresponding federal minimum wage in that year. In the CEX, we drop households that report implausibly low quarterly consumption expenditures (less than $\$ 100$, in 2000 dollars). In order to reduce measurement error, we also exclude CEX households flagged as "incomplete income reporters."
} 
We use an imputation procedure to adjust for top-coding based on the Pareto distribution. We then select households in which the male is between the ages of 25 and 59, and works at least 260 hours in the year. ${ }^{25}$ In both datasets, the hourly wage is computed as annual pre-tax labor earnings divided by annual hours worked. ${ }^{26}$ To avoid severe selection issues, we use wages and hours for males only. Our measure of household consumption includes expenditures on nondurables, services, small durables, and an estimate of the service flow from vehicles and housing. All nominal variables are deflated using the Consumer Price Index (CPI-U). Our PSID and CEX samples are updated versions of those constructed by Heathcote, Perri, and Violante (2010). We refer to that paper for a detailed description of these two surveys, the sample selection, and exact variable definitions.

As discussed in Section 3.1, we regress individual log wages, individual log hours, and household log consumption on year dummies, a quartic in age, and (for consumption) household composition dummies.

We then use the residuals from these regressions to construct variances and covariances in levels and differences for all available age/year cells constructed by grouping observations in any given year into 31 five-year overlapping age classes (27-57). ${ }^{27}$ From the PSID data we construct (i) 1,085 age/year covariances corresponding to 31 age groups over 35 years (19671996, 1998, 2000, 2002, 2004, 2006) for each of the three moments in levels involving wages and hours; (ii) 899 age/year covariances corresponding to 31 age groups over 29 years for each of the three moments in first differences; and (iii) 1,203 age/year covariances corresponding to 31 age groups over 33 years for each of the three moments in second differences. From the CEX data, we construct 837 age/year covariances corresponding to 31 age groups over 27 years (1980-2006) for each of the three moments in levels involving consumption.

\footnotetext{
${ }^{25}$ The resulting unbalanced panel from the PSID comprises 2,930 individuals and 93,153 person-year observations. The resulting repeated cross sections from the CEX have a total of 87,966 household-year observations (on average, 3,258 households per year).

${ }^{26}$ Labor earnings are defined in both surveys as the sum of all income from wages, salaries, commissions, bonuses, and overtime, and the labor component of self-employment income.

${ }^{27}$ For example, the variance of $\log$ wages for the youngest age group (age class 27 ) at date $t$ is constructed with all wage observations for individuals aged 25-29 at date $t$, the variance of log wages for the next age group (age class 28) at date $t$ is constructed with all wage observations for individuals aged 26-30 at date $t$, and similarly for all other age groups until the oldest one (age class 57). Since the number of observations in many one-year age cells is very small, this procedure reduces sampling variation. We apply the same procedure to construct the model analogue of these moments.
} 


\subsection{Estimation method}

The structural estimation of the model uses the minimum distance estimator introduced by Chamberlain (1984), which minimizes a weighted squared sum of the differences between each moment in the model and its data counterpart. Let $\mathbf{m}(\Lambda)$ denote the $(J \times 1)$ vector of theoretical covariances, and $\Lambda$ denote the $(N \times 1)$ vector of parameter values to estimate. Correspondingly, we define $\hat{\mathbf{m}}$ as the vector of empirical covariances. The estimator solves the following minimization problem:

$$
\min _{\Lambda}[\hat{\mathbf{m}}-\mathbf{m}(\Lambda)]^{\prime} \mathcal{W}[\hat{\mathbf{m}}-\mathbf{m}(\Lambda)]
$$

where $\mathcal{W}$ is a $(J \times J)$ weighting matrix. Standard asymptotic theory implies that the estimator $\widehat{\Lambda}$ is consistent and asymptotically Normal. Due to the small sample size, we make two choices: (i) we use an identity matrix for $\mathcal{W}$; ${ }^{28}$ (ii) we compute $90-10$ confidence intervals through a block-bootstrap procedure based on 500 replications. ${ }^{29}$

Proposition 2 and its corollaries indicate that, absent additional assumptions, one cannot identify various parameters at the end points of the sample or in the missing PSID survey years. We assume that prior to 1967 the variances of all shocks were equal, in each year, to their respective values in $1967 .{ }^{30}$ We describe the other minor technical identifying assumptions in Technical Appendix D. Overall, the estimation uses $J=11,532$ moment conditions for $N=232$ parameters. ${ }^{31}$

Parameters set outside the model We set $\delta=0.996$ to match the annualized probability of surviving from age 25 to age 60 for US men. ${ }^{32}$ To estimate the progressivity parameter

\footnotetext{
${ }^{28}$ The bulk of the literature follows this strategy, in light of the Monte Carlo simulations of Altonji and Segal (1996) who argue that in common applications there is a substantial small sample bias when using the optimal weighting matrix characterized by Chamberlain (1984).

${ }^{29}$ Bootstrap samples are drawn at the household level with each sample containing the same number of observations as the original sample. The implied confidence intervals thus account for arbitrary serial correlation, heteroscedasticity, and estimation error induced by the first-stage regression of individual observations on age, time, and household type.

${ }^{30}$ Alternatively, we could have treated the cumulative variances of the insurable and uninsurable components for the cohorts alive in 1967, i.e., $\left\{v_{\kappa^{a}, 1967}, v_{\alpha^{a}, 1967}\right\}_{a=27}^{57}$, as parameters to be estimated. When pursuing this alternative estimation strategy, we found the results to be virtually identical to those under the baseline "steady-state" identification scheme.

${ }^{31}$ The large number of parameters is due to the fact that we did not restrict time/cohort variation in the variances of wage shocks and preference dispersion. We have also estimated the model by restricting these variances to follow time polynomials, and we found very similar results.

${ }^{32}$ The survival rate $\delta$ does not appear in any of the age/year moments we use to estimate the model, and hence its calibration has no bearing on the parameter estimates. We use $\delta$ only to construct the aggregate cross-sectional variances and covariances plotted to measure the fit of the model against the data. The fit is extremely robust to varying $\delta$ within a plausible range.
} 
$\tau$, for each household in our PSID sample we compute disposable income as income minus federal and state taxes (calculated based on the NBER's TAXSIM program), plus government cash payments (e.g., TANF, UI benefits) and income-tested transfers in kind (e.g., food stamps). ${ }^{33}$ From equation (4), a consistent estimate of $1-\tau$ can be obtained by regressing log household disposable income on log household pre-government income, including a constant in the regression. The ordinary least squares estimate of this coefficient implies $\tau=0.265$ (s.e. $=0.001)$. The associated $R^{2}$ measure of fit is 0.88 , which demonstrates that our functional form provides a good approximation to the actual US tax system.

Around 1992 (survey year 1993), the first year of computer-assisted telephone interviewing in the PSID, individual earnings and hours appear unusually volatile. This surge in volatility has been widely attributed to a temporary increase in measurement error. We therefore assume that the variance of measurement error in earnings and hours in 1992 is twice as large as in other years. ${ }^{34}$

\section{Results}

Table 1 reports parameter estimates. Our estimates for the two preference elasticity parameters are $\gamma=1.56$ and $\sigma=1.69$. In both cases the confidence intervals are narrow. Given our assumed value for the tax progressivity parameter $\tau$, the implied tax-modified Frisch elasticity with respect to pre-tax wages is $1 / \hat{\sigma}=(1-\tau) /(\sigma+\tau)=0.38$, a value that is broadly consistent with the microeconomic evidence (see, e.g., Keane, 2011).

The average estimated values for the variances of uninsurable and insurable permanent wage shocks $\left(\overline{v_{\omega}}\right.$ and $\left.\overline{v_{\eta}}\right)$ and corresponding cohort effects $\left(\overline{v_{\alpha^{0}}}\right.$ and $\left.\overline{v_{\kappa^{0}}}\right)$ indicate that almost $40 \%$ of permanent life-cycle wage innovations are insurable, while less than $30 \%$ of initial wage variation at labor market entry is insurable. The estimated average transitory wage variance is $\overline{v_{\theta}}=0.042$, an order of magnitude larger than the variance of permanent shocks. The entire time series for these variances are reported in Table 5 in the Technical Appendix. Finally, our estimates for the variances of measurement error in log hours worked, individual earnings, and household consumption are, respectively, 0.035, 0, and 0.053. ${ }^{35}$

\footnotetext{
${ }^{33}$ Since state income taxes from TAXSIM are only available from 1978, we exclude years 1967-1977 in this calculation. See Appendix B in Heathcote, Perri, and Violante (2010) for details.

${ }^{34}$ It is straightforward the derive the new theoretical moments in levels for 1992, in first differences for 1992-1993, and second differences for 1992 and 1994.

${ }^{35}$ The estimate of zero measurement error in earnings might seem surprising. However, Gottschalk and Huynh (2010) find that the cross-sectional variance of true earnings is greater, rather than smaller, than that
} 
Table 1: Baseline Parameter Estimates

\begin{tabular}{cccccc}
\hline \hline \multicolumn{2}{c}{ Preference Elasticities } & \multicolumn{3}{c}{ Life-Cycle Shocks } \\
\hline$\sigma$ & $\gamma$ & $\overline{v_{\omega}}$ & $\overline{v_{\eta}}$ & $\overline{v_{\theta}}$ \\
1.686 & 1.555 & 0.0065 & 0.0040 & 0.042 \\
{$[1.581,1.871]$} & {$[1.502,1.641]$} & & {$[0.002,0.013]$} & {$[0.001,0.008]$} & {$[0.034,0.050]$} \\
\multicolumn{2}{r}{} & & & & \\
\multicolumn{2}{c}{ Initial Heterogeneity } & & \multicolumn{3}{c}{ Measurement Error } \\
\hline$\overline{v_{\alpha^{0}}}$ & $\overline{v_{\kappa^{0}}}$ & $\overline{v_{\varphi}}$ & $v_{\mu y}$ & $v_{\mu h}$ & $v_{\mu c}$ \\
0.109 & 0.040 & 0.517 & 0.000 & 0.035 & 0.053 \\
{$[0.093,0.123]$} & {$[0.019,0.063]$} & {$[0.448,0.625]$} & {$[0.000,0.000]$} & {$[0.034,0.036]$} & {$[0.051,0.055]$} \\
\hline
\end{tabular}

Notes: Bars denote sample averages. 90-10 bootstrapped confidence intervals are shown in parentheses.

\subsection{Life-cycle fit}

Figures 1 and 2 compare the evolution of model and data along the life-cycle dimension and show that the model-implied moments align closely with their empirical counterparts from the PSID and the CEX. In particular, the model-implied moments almost always lie within the 90-10 confidence intervals around the empirical moments. With the help of these figures, we offer some economic intuition relating the life-cycle profiles for inequality to the parameter estimates described above. We then demonstrate that each feature of the baseline model plays an important role in accounting for the empirical moments by estimating a set of restricted models.

Understanding parameter estimates In both US and model-simulated data, the variance of $\log$ wages increases by around $35 \log$ points, approximately linearly, between ages 27 and 57. In contrast, the variance of log consumption grows much less, by about $10 \log$ points over the life cycle. The much steeper life-cycle increase in wage dispersion relative to consumption dispersion explains why a significant share (38\%) of permanent shocks to wages are estimated to be insurable.

The fact that the empirical profile for the variance of log hours is fairly flat, notwithstanding the fact that dispersion in wages increases sharply as permanent shocks cumulate, points to a relatively low Frisch elasticity of labor supply. However, we show below that the model fits poorly if we impose a zero Frisch elasticity.

The point estimate for $\gamma$ exceeds one because the covariance between wages and hours is in the survey data. They argue that this reflects a non-classical structure for measurement error in earnings. 
negative, indicating significant wealth effects from uninsurable shocks to wages (recall that insurable wage shocks push this covariance up). ${ }^{36}$ The framework allows for one alternative way to generate a negative wage-hours covariance, namely measurement error in hours. However, the estimation procedure does not attribute the low covariance entirely to measurement error because this would translate into an excessively high variance for the growth of individual hours. ${ }^{37}$

Figure 2 shows that the model also accounts well for the life-cycle moments in first and second differences. ${ }^{38}$ How does the model discriminate between transitory insurable shocks and measurement error? Equations (29)-(32) illustrate that if moments in first (and second) differences were driven primarily by measurement error in hours, then the correlation between hours and wage growth would be close to minus one. A substantial amount of true transitory wage variation is needed to raise this correlation to the level observed in the data. Finally, note that the variance of biannual wage and hours growth (the bottom panels) is not much larger than the variance of annual growth, which helps explain why the estimated variances for permanent shocks are small relative to transitory shocks.

Alternative models: What goes wrong? To better understand why each model element is needed to account for the observed cross-sectional moments, we now discuss a range of experiments in which we shut down one model element at a time, and re-estimate the model. See Table 2 for the parameter estimates of these alternative models.

We first consider two alternative insurance market structures. In the first, we assume perfect private insurance against permanent life-cycle shocks, by imposing $v_{\omega t}=0$. In the second, we make the opposite assumption, namely that there is no private insurance against permanent life-cycle shocks, by imposing the restriction $v_{\eta t}=0$. This economy captures the spirit of the permanent income hypothesis (PIH), according to which transitory shocks are

\footnotetext{
${ }^{36}$ In a similar spirit, Chetty (2006) argues that existing empirical evidence on the response of hours to permanent shocks to wages can be used to bound estimates for risk aversion. An advantage of our fully structural approach is that we can identify $\gamma$ in an environment with a mix of uninsurable and insurable permanent wage shocks.

${ }^{37}$ Figure 1 indicates that the estimated model exaggerates the increase in the correlation between wages and hours observed over the life cycle. A larger value for $\gamma$ would improve the model's fit in this dimension, by amplifying the offsetting effect on hours or permanent uninsurable wage shocks. However, a larger value for $\gamma$ would also steepen the age decline in the theoretical correlation between hours and consumption. See equations (19) and (21). Thus the estimated value for $\gamma$ reflects a compromise in an attempt to reconcile various conflicting moments.

${ }^{38}$ For example, the top left and bottom left panels show the cross-sectional variances of annual and biannual log wage growth. The first differences apply to the period 1967-1996, while the second differences refer to $1967-2006$.
} 
Table 2: Parameter Estimates: Alternative Models

\begin{tabular}{rcccccc}
\hline \hline & $(1)$ & $(2)$ & $(3)$ & $(4)$ & $(5)$ & $(6)$ \\
\hline$\sigma$ & 1.686 & 2.309 & 1.730 & $\infty^{*}$ & 1.102 & 1.281 \\
$\gamma$ & 1.555 & 1.376 & 1.617 & $1.555^{*}$ & 1.563 & 2.121 \\
$v_{\mu h}$ & 0.035 & 0.036 & 0.035 & 0.031 & 0.038 & 0.035 \\
$v_{\mu y}$ & 0.000 & 0.000 & 0.000 & 0.000 & 0.000 & $0^{*}$ \\
$v_{\mu c}$ & 0.053 & 0.056 & 0.052 & 0.038 & 0.086 & 0.053 \\
$\bar{v}_{\widehat{\varphi}}$ & 0.054 & 0.053 & 0.055 & 0.057 & $0^{*}$ & 0.031 \\
$\bar{v}_{\alpha^{0}}$ & 0.110 & 0.166 & 0.085 & 0.097 & 0.101 & 0.080 \\
$\bar{v}_{\omega}$ & 0.0065 & $0^{*}$ & 0.0091 & 0.0056 & 0.0073 & 0.0077 \\
$\bar{v}_{\kappa^{0}}$ & 0.040 & 0.011 & 0.070 & 0.050 & 0.054 & 0.066 \\
$\bar{v}_{\eta}$ & 0.0040 & 0.0081 & $0^{*}$ & 0.0052 & 0.0033 & 0.0033 \\
$\bar{v}_{\theta}$ & 0.042 & 0.043 & 0.044 & 0.045 & 0.037 & 0.040 \\
\hline $1 / \widehat{\sigma}$ & 0.377 & 0.286 & 0.368 & $0^{*}$ & 0.538 & 0.475 \\
\hline$S S R$ & 9.711 & 11.928 & 14.066 & 12.331 & 12.568 & - \\
\hline
\end{tabular}

Notes: Externally set values are followed by an asterisk. The columns are (1) baseline model (see Table 1), (2) complete markets for all shocks $\left(v_{\omega t}=0\right),(3)$ no private insurance against permanent shocks $\left(v_{\eta t}=0\right),(4)$ inelastic labor supply $(\sigma \rightarrow \infty)$, (5) no preference heterogeneity $\left(v_{\varphi t}=0\right)$, and (6) baseline model without using CEX consumption data (Section 5.5). Variables with bars (e.g., $\bar{v}_{\theta}$ ) denote average estimates over time. Values for $1 / \widehat{\sigma}$ are implied by the other parameter estimates. $S S R$ is the sum of squared residuals.

largely insurable, while permanent shocks are uninsurable.

The estimated "complete markets" model $\left(v_{\omega t}=0\right)$ delivers twice as large an average variance for permanent insurable shocks relative to the baseline model. Absent changes in other parameter values, this would imply too much dispersion in hours worked and too little dispersion in consumption: thus, the estimation also delivers a larger estimate for $\sigma$ (a lower Frisch) and a higher estimate for $v_{\alpha^{0}, t}$ (more uninsurable wage dispersion at labor market entry). However, absent permanent uninsurable shocks, the model has no way to generate the observed rise in consumption dispersion over the life-cycle. Another indication that this model exaggerates insurance against life-cycle shocks is that it generates much too large an increase in the correlation between wages and hours over the life cycle.

The estimated PIH model $\left(v_{\eta t}=0\right)$ delivers similar parameter estimates to the baseline model, with the exception that the average variance of permanent uninsurable shocks rises from 0.0065 to 0.0091 . Perhaps surprisingly, the estimated model replicates fairly closely the empirical life-cycle profile for the variance of log consumption, because uninsurable wage shocks are partially smoothed via labor supply and progressive taxation. However, the 
model now generates a counterfactual decline over the life cycle in the correlation between wages and hours worked. Recall that permanent uninsurable shocks drive this correlation down, while permanent insurable shocks (shut off in this experiment) drive the correlation up. Consequently, the estimated model also delivers a life-cycle increase in the variance of earnings that is much too small.

We next experiment with shutting off flexible labor supply by setting $\sigma=\infty$ in the baseline model. ${ }^{39}$ With inelastic labor supply, measurement error is the only source of variance in the growth of individual hours. However, with a zero Frisch elasticity, measurement error in hours implies a negative correlation between wages and hours worked, while this correlation is close to zero in the data. The estimation compromises, delivering too little variation over time in individual hours and a counterfactually negative wage-hours correlation. In addition, the model with inelastic hours generates too much comovement between hours worked and consumption because it rules out income effects as a force to offset preference heterogeneity. We conclude that allowing for elastic labor supply is essential in accounting for all moments involving hours worked.

In our last experiment, we eliminate preference heterogeneity by imposing $v_{\widehat{\varphi}}=0$. In our baseline model, preference heterogeneity is required to replicate the positive empirical correlation between hours worked and consumption. Absent preference variation, the model generates a counterfactual negative correlation since, with $\gamma>1$, individuals with a higher uninsurable wage component enjoy more consumption but work fewer hours - see equations (7) and (8). Preference heterogeneity also plays an important role in generating cross-sectional dispersion in hours worked and consumption, and when it is shut down the estimation looks for alternative ways to replicate these moments. In particular, it assigns larger values for the variances of measurement error and delivers a higher Frisch elasticity.

We conclude this section by highlighting two key messages from this exploration of alternative models. First, the overall model fit worsens dramatically in each restricted version of the baseline model we estimate (see the sum of squared residuals in Table 2), indicating that each model element plays an important quantitative role in accounting for observed dynamics of inequality. In particular, the data - and especially the moments involving hours worked speak strongly to the existence of risk-sharing mechanisms that allow households to insure

\footnotetext{
${ }^{39}$ Technically, we set $\sigma=500$. With $\sigma$ large but finite, the model can still generate dispersion in hours through preference heterogeneity. Given a Frisch elasticity near zero, our identification strategy for $\gamma$ (based on cross-sectional moments involving hours) fails. Thus we set $\gamma$ equal to its value in the baseline model.
} 
a fraction (but only a fraction) of permanent idiosyncratic fluctuations in wages. They also speak strongly to the existence of two fundamental drivers of dispersion in hours worked: a positive elasticity in response to wage fluctuations and a second source of dispersion in hours that is unrelated to wages.

Second, it is important to estimate the scope for private risk sharing and preference parameters jointly. The logic is simply that both matter for the dynamics of consumption and labor supply. If we use more restricted models for private risk sharing (by imposing too much or too little private risk sharing), the estimation contorts estimates for preference elasticities or for preference heterogeneity in order to try to match the same moments involving consumption and hours. If we restrict the model for preferences (by imposing inelastic hours or an absence of preference heterogeneity), the model delivers the wrong estimate for the fraction of wage risk that is insurable.

\subsection{Insurance and inequality over the life cycle}

The first key question motivating our paper was: How insurable are life-cycle shocks to wages? And what channels mediate the transmission from wages to consumption? There are three reasons for incomplete pass-through from wages to consumption. First, labor supply decisions determine how wage shocks transmit to earnings. Second, if changes in earnings reflect shocks that are privately insurable, they will not be reflected in changes in consumption. Third, even if they reflect (privately) uninsurable shocks, the progressive tax/transfer system provides additional smoothing.

Pass-through coefficients Let $\phi_{t}^{w, c}$ denote the pass-through coefficient from wages to consumption, defined as the OLS coefficient from a panel regression of model-simulated changes in $\log$ consumption between $t-1$ and $t$ on permanent (uninsurable or insurable) changes in log individual wages. ${ }^{40}$ We focus here on permanent shocks, because transitory shocks are fully privately insurable in our framework. The elasticity of consumption with respect to an uninsurable permanent innovation $\omega_{t}$ is $(1-\tau)(1+\widehat{\sigma}) /(\widehat{\sigma}+\gamma)$ (see eq. 7 ), while consumption does not respond to permanent insurable innovations $\eta_{t}$. Thus $\phi_{t}^{w, c}$ is

\footnotetext{
${ }^{40}$ According to the model of the household described in Section 3.1, the household composition dummy $D(g, k)$ drops out when looking at the growth rate of $\log$ consumption. This implies that $\phi_{t}^{w, c}$ can be interpreted either as measuring pass-through to raw household consumption, or as pass-through to equivalized consumption.
} 
given by

$$
\phi_{t}^{w, c}=(1-\tau) \cdot \frac{1+\widehat{\sigma}}{\widehat{\sigma}+\gamma} \cdot \frac{v_{\omega t}}{v_{\omega t}+v_{\eta t}} .
$$

Plugging in the estimated values for $\gamma, \sigma, \overline{v_{\omega}}$, and $\overline{v_{\eta}}$ from Table 1 along with $\tau=0.265$ gives an average pass-through coefficient of $\bar{\phi}^{w, c}=0.395$. Thus, on average, only slightly less than $40 \%$ of a permanent wage shock transmits to consumption. ${ }^{41}$

The roles of progressive taxation, labor supply, and private risk sharing in delivering consumption smoothing against permanent wage fluctuations are captured, respectively, by the three terms in the pass-through formula for $\phi_{t}^{w, c}$. Evaluated at the sample-average parameter estimates, $38.1 \%$ of permanent wage shocks are privately insured. Of the non-insured component of wages, $13.2 \%$ of fluctuations are smoothed through labor supply, reflecting the fact that our estimate for $\gamma$ is larger than one (see eq. 8). Of the residual component transmitted to earnings, $26.5 \%$ of fluctuations are smoothed through progressive taxation. We conclude that all three channels play important roles in mediating the response of consumption to permanent wage shocks. Private insurance is the most important of these channels, followed by progressive taxation. ${ }^{42}$

While the primitive shocks in our model are shocks to wages, we can also compute a pass-through coefficient from pre-tax individual earnings to consumption:

$$
\phi_{t}^{y, c}=(1-\tau) \cdot \frac{v_{\omega t}}{v_{\omega t}+\left(\frac{\hat{\sigma}+\gamma}{\hat{\sigma}}\right)^{2} v_{\eta t}}
$$

which implies an average value of $\bar{\phi}^{y, c}=0.289$. Blundell, Pistaferri, and Preston (2008, Table 7) estimate a quantitatively similar pass-through coefficient of 0.225 from permanent shocks to male earnings to non-durable consumption on US data. They conclude that the bulk of permanent income risk is insurable. Our framework suggests that one has to be cautious with this interpretation for two reasons. First, earnings are endogenous in the model, and the pass-through from the primitive wage shocks to consumption is almost 40 percent larger than the one for earnings. Second, low estimated pass-through from earnings to consumption

\footnotetext{
${ }^{41}$ The pass-through coefficients implied by the restricted models described in Section 5.1 are of a similar magnitude. The logic is that when one insurance mechanism is shut down, the model attributes a greater smoothing role to other channels, so as to replicate the same empirical moments. For example, with inelastic labor supply $\bar{\phi}^{w, c}=0.38$, while without progressive taxation $\bar{\phi}^{w, c}=0.36$.

${ }^{42} \mathrm{An}$ alternative way to gauge the roles of these different insurance mechanisms is to shut them off one at a time, and then compute by how much the implied pass-through coefficient would increase, holding constant other parameter values. We implement this by setting, respectively, $\overline{v_{\eta}}=0, \sigma \rightarrow \infty$, and $\tau=0$, in which cases $\bar{\phi}^{w, c}$ rises from 0.39 to, respectively, 0.64, 0.46, and 0.51. In this second calculation, the ranking of insurance channels is thus the same as in the first one.
} 
does not necessarily indicate that the underlying shocks are mostly insurable in nature. To see this, consider the extreme case in which preferences are linear in hours worked $(\sigma=0)$. The pass-through coefficient from earnings to consumption would suggest perfect smoothing, i.e., $\lim _{\sigma \rightarrow 0} \phi_{t}^{y, c}=0$, irrespective of the size of $v_{\omega t}$ and $v_{\eta t}{ }^{43}$

Growth in life-cycle variances An alternative, and more common, metric for quantifying the extent of insurance against life-cycle income fluctuations is to compare the withincohort life-cycle growth in the variances of consumption on the one hand, and wages or earnings on the other (see, e.g., Blundell and Preston, 1998; Storesletten, Telmer, and Yaron, 2004a; Huggett, Ventura, and Yaron, forthcoming). The analytical expressions for these moments are in equations (23) and (26).

Our framework uncovers a useful relationship between the ratio of life-cycle growth in the variance of consumption to growth in the variance of wages on the one hand, and the pass-through coefficient described above on the other. Assuming $\Delta v_{\theta t}=0$, we obtain

$$
\frac{\Delta \operatorname{var}_{t}^{a}(\log \hat{c})}{\Delta v a r_{t}^{a}(\log \hat{w})}=(1-\tau)^{2}\left(\frac{1+\hat{\sigma}}{\hat{\sigma}+\gamma}\right)^{2} \frac{v_{\omega t}}{v_{\omega t}+v_{\eta t}}=(1-\tau)\left(\frac{1+\hat{\sigma}}{\hat{\sigma}+\gamma}\right) \cdot \phi_{t}^{\omega, c} .
$$

This relation reveals that these two alternative measures of insurance coincide exactly if and only if progressive taxation and labor supply are both absent as smoothing mechanisms, i.e., when either (i) $\tau=0$ and $\sigma \rightarrow \infty$, or (ii) $\tau=0$ and $\gamma=1$. In the latter case, even though labor supply is elastic, it is not used to smooth uninsurable shocks to wages.

If $\tau>0$ or if $\gamma>1$ (and $\sigma<\infty$ ), then insurance provided through taxation and/or labor supply shows up more strongly in the ratio $\Delta \operatorname{var}_{t}^{a}(\log \hat{c}) / \Delta \operatorname{var}_{t}^{a}(\log \hat{w})$ than in the pass-through coefficient $\phi_{t}^{\omega, c}$. At our baseline parameter values, the life-cycle increase in the variance of $\log$ consumption is only $25 \%$ of the corresponding increase in the variance of $\log$ wages, even though around $40 \%$ of permanent wage shocks transmit to consumption.

\subsection{Insurance and inequality over time}

Insurability over time Table 5 in the Technical Appendix contains the complete set of year-by-year estimates for all the time-varying parameters of the model. Figure 3 summarizes

${ }^{43}$ We can also define a pass-through coefficient from permanent wage changes to pre-tax earnings:

$$
\phi_{t}^{w, y}=\frac{1+\widehat{\sigma}}{\widehat{\sigma}+\gamma} \cdot \frac{v_{\omega t}}{v_{\omega t}+v_{\eta t}}+\frac{1+\widehat{\sigma}}{\widehat{\sigma}} \cdot \frac{v_{\eta t}}{v_{\omega t}+v_{\eta t}} .
$$

In our model, $\phi_{t}^{w, c}=\phi_{t}^{w, y} \cdot \phi_{t}^{y, c}$ if and only if either (i) $v_{\eta t}=0$, (ii) $\gamma=0$, or (iii) $\sigma \rightarrow \infty$. 
what these estimates imply for changes over time in the structure of relative wages. Panel A shows that the variance of the total uninsurable component $\left(\alpha_{t}\right)$ declines in the 1970s and then rises in the remainder of the sample period. This pattern broadly accords with the fall of the skill premium in the late 1960s to mid-1970s, and the subsequent increase in the 1980s and beyond. Under this interpretation, "skill-biased demand shifts" represent an important source of uninsurable wage shocks. ${ }^{44}$ The total cross-sectional variance of the permanent insurable component of wages $\left(\kappa_{t}\right)$ is generally increasing throughout the first two decades, but declines somewhat from the mid-1980s (Panel B). The variance of transitory insurable shocks $\left(\theta_{t}\right)$ plotted in Panel $\mathrm{C}$ grows steadily throughout the sample, consistent with Moffitt and Gottschalk's (2002) estimates for earnings dynamics.

Combining these estimates allows us to address the second of our motivating questions: What fraction of the observed rise in wage dispersion over our sample period was privately insurable for US households? Panel D of Figure 3 indicates that in 1967 the insurable component of wages accounted for around $25 \%$ of the cross-sectional variance of log wages, while by the early 1980s this fraction was around 45\%. Since then, the variances of the insurable and uninsurable components of wages have risen at a similar rate, leaving the fraction of wage fluctuations insured relatively stable.

Finally, note that the "cohort" plots in Panels A and B are rather steady over time, indicating that the bulk of the dynamics in cross-sectional wage dispersion reflect changes in the variances of life-cycle shocks.

Time series fit The variance of log male wages increases by around 15 log points over the sample period, with especially rapid growth in the 1980s. We now turn to explaining how the moments involving consumption and hours account for the partition of this rise between larger insurable versus larger uninsurable shocks described in Figure 3. Figure 4 plots the evolution over time of these moments, alongside the corresponding values for the estimated model.

Over the first half of the sample, we see a sharp rise in the wage-hours correlation (Panel D). The model interprets this as indicating a rise in the variance of the insurable wage component and a fall in the variance of the uninsurable component. The latter translates into a theoretical prediction of modestly declining consumption inequality before 1980, when

\footnotetext{
${ }^{44}$ This interpretation is consistent with Attanasio and Davis (1996) and with Heathcote, Storesletten, and Violante (2010b), who, in the context of an augmented version of the standard incomplete-markets model, show that skill-biased demand shifts are the main driver of the rise in consumption inequality.
} 
our CEX sample begins. This pattern for consumption inequality parallels the dynamics of the skill premium over the period. ${ }^{45}$

After 1980 consumption data are available and further inform the estimation. The variance of log consumption grows by only about 5 log points between 1980 and 2006, in line with earlier estimates by Krueger and Perri (2006). This rise, paired with the one in the wage-consumption correlation, calls for an increase in uninsurable wage dispersion and a slowdown in the rise of insurable wage dispersion. This pattern is also consistent with the end of growth in the empirical wage-hours correlation. The increase in the variance of consumption over time is small relative to the increase in uninsurable wage dispersion (see Figure 3) because, as with the life-cycle dimension, labor supply and progressive taxation mitigate the impact of uninsurable wage dispersion on consumption dispersion.

Larger uninsurable wage shocks tend to drive the consumption-hours correlation down over time. To offset this force and replicate the roughly flat pattern for the correlation in the data, the estimation calls for a modest increase over time in preference dispersion (see Table 5 in the Technical Appendix).

Figure 5 shows the time series plots for moments in first and second differences. Recall that these moments are driven primarily by measurement error and transitory wage shocks, given the relatively small estimated variances for the innovations to permanent shocks. Thus we can point to the rise in the variance of wage growth over time as the source of the corresponding rise in the estimated variance of transitory shocks (Panel C of Figure 3). These larger transitory shocks, in turn, account for the model-predicted increase in the correlation between wage and hours growth. ${ }^{46}$

\subsection{Inequality decomposition}

We now turn to the third motivating question of our paper. Is observed cross-sectional inequality primarily the result of life-cycle shocks, initial heterogeneity in productivity and preferences, or simply measurement error? Given parameter estimates and the moment expressions in eqs. (17)-(22), variance decompositions are unique and easy to compute.

\footnotetext{
${ }^{45}$ It is also broadly consistent with evidence from Slesnick (2001, chapter 6), who, notwithstanding data comparability issues, uses CEX data pre-1980 in order to construct a longer series for US consumption dispersion. Guvenen and Smith (2010, Figure A.1) impute nondurable consumption into the PSID from the CEX going back to 1967 and also uncover a decline in the variance of log consumption over the first decade of their sample.

${ }^{46}$ Recall that we assumed measurement error in hours doubled in 1992, the first year of a new PSID survey methodology. This allows us to match the spike in the variance of wage and hours growth in the early 1990 s.
} 
Table 3: Decomposition of Cross-Sectional Inequality

\begin{tabular}{|c|c|c|c|c|c|c|c|}
\hline & \multirow{3}{*}{$\begin{array}{c}\text { Total } \\
\text { Variance }\end{array}$} & \multicolumn{6}{|c|}{ Percent Contribution to Total Variance } \\
\hline & & Initial & Heteroge & aeity & Life-Cy & Shocks & Measurement \\
\hline & & Prefs. & Unins. & Ins. & Unins. & Ins. & Error \\
\hline $\operatorname{var}(\log \hat{w})$ & 0.351 & 0.0 & 32.0 & 8.3 & 21.7 & 28.2 & 9.9 \\
\hline $\operatorname{var}(\log \hat{h})$ & 0.107 & 46.6 & 1.9 & 4.0 & 1.3 & 13.5 & 32.8 \\
\hline $\operatorname{var}(\log \hat{y})$ & 0.432 & 11.1 & 22.8 & 10.1 & 13.1 & 42.9 & 0.0 \\
\hline $\operatorname{var}(\log \hat{c})$ & 0.159 & 16.9 & 29.5 & 0.0 & 20.0 & 0.0 & 33.5 \\
\hline
\end{tabular}

Sample average In Table 3, we report the average contribution of each component across the entire $1967-2006$ period. $^{47}$

Interestingly, initial heterogeneity explains between $40 \%$ and $50 \%$ of the observed variance for all variables. However, the source of this inequality at labor market entry varies. Preference heterogeneity is dominant in accounting for dispersion in hours worked, whereas heterogeneity in productivity (mostly uninsurable) is paramount for wages, earnings, and consumption. Measurement error also plays a large role, accounting for one-third of the observed variance for both hours and consumption. The flipside of the finding that initial heterogeneity and measurement error account for a large share of dispersion in consumption and hours worked is that life-cycle shocks to wages contribute relatively little to dispersion in these variables. Instead, life-cycle shocks explain half of the cross-sectional variation in wages and earnings.

We conclude that there is no simple answer to the question: What determines measured inequality among households? The answer depends on the variable of interest: for hours it is mostly preference heterogeneity and measurement error; for wages and earnings it is dispersion in productivity, predominantly over the life cycle; while for consumption it is a mix of all these factors.

Change over time Table 4 decomposes growth in variances over time into components attributable to changes in uninsurable and insurable wage dispersion and changes in preference dispersion. Because there is a fair amount of year-to-year variation in the estimated shock variances, we compare the average variances over the 1967-1971 period to those over the 2002-2006 period.

\footnotetext{
${ }^{47}$ These values are computed by taking survival-probability-weighted averages across within-age-group values for dispersion at each date, and then computing a simple average across the years in our sample.
} 
Table 4: Decomposition of Changes in Inequality over Time

\begin{tabular}{lcccc}
\hline \hline & Total Growth & \multicolumn{3}{c}{ Percent } \\
& 2002-6 vontribution of \\
\cline { 2 - 5 } & & $v_{\varepsilon}$ & $v_{\alpha}$ & $v_{\widehat{\varphi}}$ \\
$\Delta \operatorname{var}(\log \hat{w})$ & 0.150 & 65.4 & 34.6 & 0.0 \\
$\Delta \operatorname{var}(\log \hat{h})$ & 0.020 & 70.7 & 4.6 & 24.8 \\
$\Delta \operatorname{var}(\log \hat{y})$ & 0.230 & 80.9 & 17.0 & 2.1 \\
$\Delta \operatorname{var}(\log \hat{c})$ & 0.024 & 0.0 & 88.9 & 11.1 \\
\hline
\end{tabular}

The table indicates that two-thirds of the overall increase in cross-sectional wage dispersion was insurable in nature. Both labor supply data (the strong growth in the wage-hours correlation) and consumption data (the modest rise in the variance of log consumption) point in this direction. For earnings, insurable shocks were even more important, accounting for $81 \%$ of the increase in the variance. As discussed previously, labor supply amplifies the effect of insurable wage shocks on earnings and mitigates the effect of uninsurable shocks.

Table 3 documented that preference dispersion explains a large share of the level of observed dispersion in hours and consumption. In contrast, Table 4 shows that changes in observed dispersion over this period are primarily driven by shifts in the structure of relative wages, while changes in preference dispersion across cohorts play a minor role. In particular, bigger insurable wage shocks explain most of the increase over time in crosssectional dispersion in hours worked, while larger uninsurable shocks account for almost $90 \%$ of the model-predicted increase in consumption dispersion.

\subsection{Estimation without consumption data}

Section 3 documents that moments involving labor supply are informative about risk sharing. Proposition 3 proves that the model is in fact identified without any data on consumption. In this section, we exploit this identification result and re-estimate the model using only data on wages and hours from the PSID. ${ }^{48}$ One motivation for this exercise is that there is some debate about how much consumption inequality has risen over time in the United States (e.g., Attanasio, Battistin, and Ichimura, 2007; Aguiar and Bils, 2011). A second motivation is that the literature on risk sharing to date focuses almost exclusively on moments involving consumption, and we would like to know whether moments involving labor supply tell a

\footnotetext{
${ }^{48}$ The identification proof of Proposition 3 is up to an external estimate for measurement error in earnings. We therefore impose the baseline estimate $v_{\mu y}=0$.
} 
similar story in terms of the fraction of idiosyncratic risk that households can insure.

When we estimate the model without CEX data, we find that the estimations with and without consumption data deliver very similar dynamics for the insurability of wage risk. Panel A of Figure 6 shows that the insurable fraction of total cross-sectional wage dispersion, as estimated without consumption data, is very close to the corresponding fraction in the baseline when consumption moments are used. Moreover, the estimated pass-through coefficient $\bar{\phi}^{w, c}$ is essentially unchanged relative to the baseline case (0.38 compared to 0.40$)$.

The main difference relative to the baseline estimates is that estimated preference heterogeneity is now much smaller (see column (6) in Table 2). Figure 6 shows that lower preference heterogeneity translates to predicted levels for the variance of log consumption (Panel B) and the consumption-hours correlation (Panel D) that are much too low relative to their empirical counterparts. We conclude that it is consumption moments, and especially the positive covariance between consumption and hours, that offer the strongest evidence of extensive preference heterogeneity. Because the model without consumption data estimates a smaller role for preference heterogeneity, it calls for a higher Frisch elasticity of labor supply $(1 / \widehat{\sigma}$ is now 0.48$)$ in order to replicate observed hours dispersion. Although the model is estimated without consumption data, it replicates the dynamics of consumption moments remarkably well, subject to the caveat about levels discussed above (see also Figures 7 and 8 in the Technical Appendix). ${ }^{49}$

Taken together, these results indicate that moments involving labor supply and moments involving consumption paint a very consistent picture with respect to how much insurance households achieve against idiosyncratic risk. This finding is reassuring from the standpoint of theory and strengthens the case for using labor supply moments in future studies of risk sharing - especially given the high quality and long panel dimension of existing datasets that record hours worked.

\section{Conclusion}

In this paper, we have developed a novel theoretical framework to analyze consumption and labor supply in the presence of idiosyncratic labor income shocks. A distinguishing feature of the model is that it can be solved analytically. Tractability is achieved by extending

\footnotetext{
${ }^{49}$ We experimented with estimating the model without consumption data while imposing the baseline estimates for $\gamma, \sigma$, and $v_{\varphi}$. In this case, the no-consumption-data model consumption moments are virtually indistinguishable from those of the baseline model.
} 
the environment of Constantinides and Duffie (1996) to incorporate flexible labor supply, partially insurable wage risk, progressive taxation, and heterogeneity in the taste for leisure. From the closed-form equilibrium allocations, it is straightforward to derive expressions for the cross-sectional (co-)variances of wages, hours, and consumption. These expressions allow, in turn, a formal identification proof and facilitate the estimation of the structural parameters. We used this framework (i) to measure the extent to which US households can insure against wage risk, (ii) to quantify how risk sharing has changed over the past 40 years -a period of sharp widening in the wage distribution, and (iii) to decompose the sources of cross-sectional inequality in wages, hours and consumption.

In our model, the population is partitioned into groups or islands. Within the island to which they belong, agents are free to trade unrestricted state-contingent claims. Across islands though, agents cannot trade claims that would allow them to pool island-level shocks. A full theoretical micro-foundation for this market structure is beyond the scope of this paper. Future work should explore whether differential information frictions associated with trade within versus between islands is a viable micro-foundation. In particular, suppose there is perfect within-island information about shocks and insurance contracts, but that neither individual shocks nor individual insurance arrangements can be observed across islands (as in Cole and Kocherlakota, 2001; and Ales and Maziero, 2011). The first assumption immediately delivers full insurance within islands. The second may make it impossible to improve insurance of island-level shocks beyond what can be achieved through trade in a risk-free bond.

The framework can be extended to shed light on a range of macroeconomic questions where heterogeneity and risk are central to the analysis.

In Heathcote, Storesletten, and Violante (2010a), we use a version of the model to explore the optimal degree of progressivity in the tax schedule, focusing on how the optimal degree of public redistribution varies with the fraction of wage risk that can be insured privately, the desire for public goods, and the elasticity of labor supply. In Heathcote, Storesletten, and Violante (2011a), we extend the model to incorporate an education choice, and quantify the welfare effect of the observed increase in the college premium, alongside the observed rise in wage risk within education groups. The framework can also be extended to incorporate a participation decision along the extensive margin. For example, with a minimum requirement on hours worked per period, each island will choose an island-specific cutoff such that agents with low realizations of $\varepsilon_{t}$ do not work. Finally, it is also possible to introduce aggregate 
shocks that are correlated with the variance of idiosyncratic risk, as in Constantinides and Duffie (1996) and Storesletten, Telmer, and Yaron (2004b), and non time-separable EpsteinZin preferences. Such an extended setup is a natural environment for studying asset pricing, and the welfare costs of business cycles.

Many of these issues have been extensively explored using conventional incompletemarkets models and numerical solution methods. The reason to revisit them is that our framework remains tractable when extended along these dimensions, making the economic forces at play transparent and readily quantifiable. 


\section{References}

Abowd, J., And D. CARD (1989): "On the Covariance Structure of Earnings and Hours Changes," Econometrica, 57(2), 411-445.

Aguiar, M., And M. Bils (2011): "Has Consumption Inequality Mirrored Income Inequality?," NBER Working Paper 16807.

Ales, L., And P. Maziero (2009): “Accounting for Private Information,” Mimeo.

Ales, L., And P. Maziero (2011): "Non-Exclusive Dynamic Contracts: Competition and the Limits of Insurance," Mimeo.

Altonji, J., And L. M. Segal (1996): "Small-Sample Bias in GMM Estimation of Covariance Structures," Journal of Business and Economic Statistics, 14(3), 353-366.

Angeletos, G.-M. (2007): "Uninsured Idiosyncratic Investment Risk and Aggregate Saving," Review of Economic Dynamics, 10(1), 1-30.

Attanasio, O., E. Battistin, and H. Ichimura (2007): "What Really Happened to Consumption Inequality in the United States?," in Hard-to-Measure Goods and Services: Essays in Honor of Zvi Griliches, ed. by E. R. Berndt, and C. R. Hulten, vol. 67 of Studies in Income and Wealth, chap. 17, pp. 515-544. University of Chicago Press.

Attanasio, O., and S. J. Davis (1996): "Relative Wage Movements and the Distribution of Consumption," Journal of Political Economy, 104(6), 1227-1262.

Attanasio, O., and N. Pavoni (2011): "Risk Sharing in Private Information Models with Asset Accumulation: Explaining the Excess Smoothness of Consumption," Econometrica, 79(4), 1027-1068.

Attanasio, O., and J.-V. Ríos-Rull (2000): "Consumption Smoothing in Island Economies: Can Public Insurance Reduce Welfare?," European Economic Review, 44(7), $1225-1258$.

Benabou, R. (2002): "Tax and Education Policy in a Heterogeneous-Agent Economy: What Levels of Redistribution Maximize Growth and Efficiency?," Econometrica, 70(2), 481-517.

Blundell, R., L. Pistaferri, and I. Preston (2008): "Consumption Inequality and Partial Insurance," American Economic Review, 98(5), 1887-1921.

Blundell, R., And I. Preston (1998): "Consumption Inequality and Income Uncertainty," Quarterly Journal of Economics, 113(2), 603-640.

Caballero, R. (1990): "Consumption Puzzles and Precautionary Savings," Journal of Monetary Economics, 25(1), 113-136. 
Campbell, J. Y., and A. Deaton (1989): "Why Is Consumption So Smooth?," Review of Economic Studies, 56(3), 357-373.

Chamberlain, G. (1984): "Panel Data," in Handbook of Econometrics, ed. by Z. Griliches, and M. Intriligator, vol. 3, pp. 1247-1318. New York: North-Holland.

Chetty, R. (2006): "A New Method of Estimating Risk Aversion," American Economic Review, 96(5), 1821-1834.

Cole, H., and N. Kocherlakota (2001): "Efficient Allocations with Hidden Income and Hidden Storage," Review of Economic Studies, 68(3), 523-542.

Constantinides, G. M., And D. Duffie (1996): "Asset Pricing with Heterogeneous Consumers," Journal of Political Economy, 104(2), 219-240.

Cunha, F., J. J. Heckman, and S. Navarro (2005): "Separating Uncertainty from Heterogeneity in Life-Cycle Earnings," Oxford Economic Papers, 57(2), 191-261.

Davis, S. J. (2003): "Comment on Krueger and Perri: On the Welfare Consequences of the Increase in Inequality in the United States," NBER Macroeconomics Annual, 18, 121-132.

Deaton, A. (1997): The Analysis of Household Surveys. Johns Hopkins University Press.

Gottschalk, P., And M. Huynh (2010): "Are Earnings Inequality and Mobility Overstated? The Impact of Nonclassical Measurement Error," Review of Economics and Statistics, 92(2), 302-315.

Guvenen, F., And A. A. Smith (2010): "Inferring Labor Income Risk from Economic Choices: An Indirect Inference Approach," Mimeo.

Heathcote, J., F. Perri, and G. L. Violante (2010): "Unequal We Stand: An Empirical Analysis of Economic Inequality in the United States, 1967-2006," Review of Economic Dynamics, 13(1), 15-51.

Heathcote, J., K. Storesletten, and G. L. Violante (2009): "Quantitative Macroeconomics with Heterogeneous Households," Annual Review of Economics, 1(1), 319-354.

- (2010a): "Redistributive Taxation in a Partial-Insurance Economy," Mimeo.

(2010b): "The Macroeconomic Implications of Rising Wage Inequality in the United States," Journal of Political Economy, 118(4), 681-722.

(2011a): "From Wages to Welfare: Decomposing Gains and Losses from Rising Inequality," Mimeo.

Mimeo. 
Huggett, M., G. Ventura, and A. Yaron (forthcoming): "Sources of Lifetime Inequality," American Economic Review.

Jappelli, T., And L. Pistaferri (2010): "The Consumption Response to Income Changes," Annual Review of Economics, 2(1), 479-506.

Kaplan, G., and G. L. Violante (2010): "How Much Consumption Insurance Beyond Self-Insurance?," American Economic Journal: Macroeconomics, 2(4), 53-87.

Keane, M. P. (2011): "Labor Supply and Taxes: A Survey," Journal of Economic Literature, 49(4), 961-1075.

Keane, M. P., And K. I. Wolpin (1997): "The Career Decisions of Young Men," Journal of Political Economy, 105(3), 473-522.

Krebs, T. (2003): "Human Capital Risk and Economic Growth," Quarterly Journal of Economics, 118(2), 709-744.

Krueger, D., And F. Perri (2006): "Does Income Inequality Lead to Consumption Inequality? Evidence and Theory," Review of Economic Studies, 73(1), 163-193.

MaCurdy, T. E. (1982): "The Use of Time Series Processes to Model the Error Structure of Earnings in a Longitudinal Data Analysis," Journal of Econometrics, 18(1), 83-114.

Moffitt, R., and P. Gottschalk (2002): "Trends in the Transitory Variance of Earnings in the United States," Economic Journal, 112(3), C68-C73.

Slesnick, D. T. (2001): Consumption and Social Welfare: Living Standards and Their Distribution in the United States. Cambridge University Press.

Storesletten, K., C. I. Telmer, and A. Yaron (2004a): "Consumption and Risk Sharing Over the Life Cycle," Journal of Monetary Economics, 51(3), 609-633.

(2004b): "Cyclical Dynamics in Idiosyncratic Labor-Market Risk," Journal of Political Economy, 112(3), 695-717.

Swanson, E. T. (forthcoming): "Risk Aversion and the Labor Margin in Dynamic Equilibrium Models," American Economic Review.

Uhlig, H. (1996): "A Law of Large Numbers for Large Economies," Journal of Economic Theory, 8(1), 41-50. 


\section{A Appendix for Publication}

\section{A.1 Proof of Proposition 1}

The proof is in two parts. In the first we describe a planner's problem and show that the solution to this problem is the allocations for consumption and hours described in Proposition 1, part (ii). In the second, we decentralize these allocations in a competitive equilibrium and show that the asset prices described in Proposition 1, part (iii), and the no-inter-island-trade result described in part (i) form part of this decentralization. In what follows, we omit some technical details the proof. See Technical Appendix A for a complete derivation.

Planner's allocations: We first solve for equilibrium allocations for consumption and hours worked by solving a set of static planning problems. Each island-level planner maximizes equally weighted period utility for a set of agents that share a common age $a$, a common preference weight $\varphi$, and a common wage component $\alpha_{t}$. Let $x_{t}=\left(a, \varphi, \alpha_{t}\right)$ denote these island-level components of the individual state. Each island-level planner controls a set of agents with the age-specific population distributions for the wage components $F_{\kappa, t}^{a}$ and $F_{\theta, t}$. Let $F_{\varepsilon, t}^{a}$ denote the implied agespecific distribution over $\kappa_{t}+\theta_{t}$. The planner's problem on an island defined by $x_{t}$ is to choose functions $c_{t}\left(x_{t}, \varepsilon_{t}\right), h_{t}\left(x_{t}, \varepsilon_{t}\right)$ to solve

$$
\max _{\left\{c_{t}\left(x_{t}, \cdot\right), h_{t}\left(x_{t}, \cdot\right)\right\}} \int\left[\frac{c_{t}\left(x_{t}, \varepsilon_{t}\right)^{1-\gamma}-1}{1-\gamma}-\exp (\varphi) \frac{h_{t}\left(x_{t}, \varepsilon_{t}\right)^{1+\sigma}}{1+\sigma}\right] d F_{\varepsilon, t}^{a}
$$

subject to the island-level resource constraint

$$
\int\left[\lambda\left(\exp \left(\alpha_{t}+\varepsilon_{t}\right) h_{t}\left(x_{t}, \varepsilon_{t}\right)\right)^{1-\tau}-c_{t}\left(x_{t}, \varepsilon_{t}\right)\right] d F_{\varepsilon, t}^{a}=0
$$

Combine the first-order conditions with respect to $c_{t}$ and $h_{t}$ to get

$$
h_{t}\left(x_{t}, \varepsilon_{t}\right)=((1-\tau) \lambda)^{\frac{1}{\sigma+\tau}} c_{t}\left(x_{t}, \varepsilon_{t}\right)^{-\frac{\gamma}{\sigma+\tau}} \exp \left(\left(\alpha_{t}+\varepsilon_{t}\right)\left(\frac{1-\tau}{\sigma+\tau}\right)-\frac{1}{\sigma+\tau}-\frac{\varphi}{\sigma+\tau}\right) .
$$

Substituting (A2) into (A1), using the definition for the tax-modified Frisch elasticity $\widehat{\sigma}=(\sigma+$ $\tau) /(1-\tau)$, and rearranging yields the expressions for $c_{t}$ and $h_{t}$ in eq. (7)-(8), where $C_{t}^{a}$ and $H_{t}^{a}$ are constants common to all agents of age $a$ in year $t$ given by

$$
\begin{aligned}
\mathcal{C}_{t}^{a} & =\frac{1}{\widehat{\sigma}+\gamma}((1+\widehat{\sigma}) \log \lambda+\log (1-\tau))+\mathcal{M}_{t}^{a} \\
\mathcal{H}_{t}^{a} & \equiv \frac{1}{(1-\tau)(\widehat{\sigma}+\gamma)}((1-\gamma) \log \lambda+\log (1-\tau))-\frac{\gamma}{\widehat{\sigma}(1-\tau)} \mathcal{M}_{t}^{a} \\
\mathcal{M}_{t}^{a} & =\frac{\widehat{\sigma}}{\widehat{\sigma}+\gamma} \log \int \exp \left(\frac{(1-\tau)(1+\widehat{\sigma})}{\widehat{\sigma}} \varepsilon_{t}\right) d F_{\varepsilon, t}^{a} .
\end{aligned}
$$

Decentralization (prices): To decentralize the solution to the above planner's problem, we start by conjecturing prices in this equilibrium. Pre-tax wages equal individual labor productivity, $w\left(x_{t}, \varepsilon_{t}\right)=\exp \left(\alpha_{t}+\varepsilon_{t}\right)$. At this wage, the intratemporal first-order condition from the agent's problem described in Section 2.1 is identical to the intra-temporal first order condition for the planner described in eq. (A2). Thus at competitive wages and the conjectured allocations (eqs. 7 and 8), agents are optimizing on the intra-temporal margin. At first blush this might seem surprising, given the presence of progressive earnings taxation in the economy. Recall, however, 
that individual agents (in the competitive equilibrium) and island-level planners (in the problem described above) are both atomistic and take the tax/transfer system parameters as exogenous.

To conjecture equilibrium prices for intertemporal insurance claims, it is convenient to revert to history-dependent notation and write $c_{t}\left(s^{t}\right)$ rather than $c_{t}\left(x_{t}, \varepsilon_{t}\right)$. We begin with the price of within-island insurance $Q_{t}\left(S ; s^{t}\right)$. The intertemporal first-order condition from the agent's problem (Section 2.1) defines the price at which an agent of age $a$ with history $s^{t}$ is willing, on the margin, to buy or sell a set of insurance contracts $B_{t}\left(S ; s^{t}\right)$ that pay $\delta^{-1}$ units of consumption if and only if $s_{t+1}=\left(\omega_{t+1}, \eta_{t+1}, \theta_{t+1}\right) \in S \subseteq S$. This price is simply the average marginal rate of substitution in those states. Substituting in the expression for consumption (7) yields the expression for $Q_{t}\left(S ; s^{t}\right)$ in eq. (9) of Proposition 1. Thus the prices $Q_{t}\left(S ; s^{t}\right)$ are consistent with optimization on the consumer side.

Note that $Q_{t}\left(S ; s^{t}\right)=Q_{t}(S)$ : insurance prices are independent of the individual history $s^{t}$ and age $a$. From eq. (9) there are two pieces to this result. First, $F_{s, t+1}$, the joint distribution over $s_{t+1}=\left(\omega_{t+1}, \eta_{t+1}, \theta_{t+1}\right)$ at $t+1$, is independent of $s^{t}$ and thus the second term in eq. (9) is independent of $s^{t}$. Second, insurance prices are also independent of age $a$, because the growth in average consumption $\exp \left(\mathcal{C}_{t+1}^{a+1}-\mathcal{C}_{t}^{a}\right)$ is independent of age, reflecting the permanent-transitory model for individual productivity dynamics. Note also that due to full insurance against $\left(\eta_{t+1}, \theta_{t+1}\right)$, the price of insurance against $\eta_{t+1}$ and $\theta_{t+1}$ simply reflects probabilities, while the price of insurance against $\omega_{t+1}$ also reflects the conditional marginal rate of substitution, with insurance against low $\omega_{t+1}$ realizations being more expensive than equally likely high $\omega_{t+1}$ realizations.

We now turn to the price function for insurance claims traded across islands. Because any contract that can be traded between islands can also be traded within an island, the inter-island price for a claim that pays $\delta^{-1}$ units of consumption iff $s_{t+1} \in Z$ must, by arbitrage, equal the corresponding within-island price, for any $Z$. This implies $Q_{t}^{*}\left(Z ; s^{t}\right)=\operatorname{Pr}\left(\left(\eta_{t+1}, \theta_{t+1}\right) \in Z\right) \times$ $Q_{t}(S)=Q_{t}^{*}(Z)$, where $Q_{t}(\mathbb{S})$ is the price of insurance against all states (i.e., a risk-free bond). Thus these prices are just probabilities times $Q_{t}(\mathbb{S})$.

Decentralization (asset purchases): We now derive asset purchases, $B_{t}\left(s_{t+1} ; s^{t}\right)$ and $B_{t}^{*}\left(\eta_{t+1}, \theta_{t+1} ; s^{t}\right)$ and verify that agents' budget constraints are satisfied in equilibrium.

Given that any available inter-island insurance contract can be purchased at the same price on the within-island market, $B_{t}^{*}\left(\eta_{t+1}, \theta_{t+1} ; s^{t}\right)=0$ for all $\left(\eta_{t+1}, \theta_{t+1}\right)$ is consistent with individual optimization (Proposition 1, part (iii)). Thus, agents are optimizing by purchasing all their insurance on the island on which they are located. At the same time, because $Q_{t}^{*}\left(Z ; s^{t}\right)=Q_{t}^{*}(Z)$, no agent has an incentive to try to sell insurance to an agent located on another island. To understand this, note that the price at which one agent (say agent $i_{1}$ ) with history $s_{i_{1}}^{t}$ is willing to buy, on the margin, a set of claims that pay if and only if $\left(\eta_{t+1}, \theta_{t+1}\right) \in Z$ is the probability of that event times agent $i_{1}$ 's expected marginal rate of substitution, i.e., $\operatorname{Pr}\left(\left(\eta_{t+1}, \theta_{t+1}\right) \in Z\right) \times Q_{t}\left(\mathbb{S} ; s_{i_{1}}^{t}\right)$. The price at which a second agent on a different island (agent $i_{2}$ with history $s_{i_{2}}^{t}$ ) is willing to sell this insurance to agent $i_{1}$ is the same probability times agent $i_{2}$ 's expected marginal rate of substitution, $\operatorname{Pr}\left(\left(\eta_{t+1}, \theta_{t+1}\right) \in Z\right) \times Q_{t}\left(\mathbb{S} ; s_{i_{2}}^{t}\right)$. If agents $i_{1}$ and $i_{2}$ did not share the same marginal rate of substitution (i.e., if $Q_{t}\left(\mathbb{S} ; s_{i_{1}}^{t}\right) \neq Q_{t}\left(\mathbb{S} ; s_{i_{2}}^{t}\right)$ ), then there could be no equilibrium without inter-island trade, because any such equilibrium would feature unexploited gains from trade. Thus, $Q_{t}\left(S, s^{t}\right)=Q_{t}(S)$ is the crucial result supporting an absence of inter-island trade.

Finally, we now derive an expression for purchases of state-contingent claims, $B_{t}\left(s_{t+1} ; s^{t}\right)$, and verify budget balance. Given $B_{t}^{*}\left(Z ; s^{t}\right)=0 \forall Z, \forall s^{t}$, realized wealth at $s^{t}$ implicitly defines insurance purchases $B_{t-1}\left(s_{t} ; s^{t-1}\right)=\delta d_{t}\left(s^{t}\right)$. Since insurance payouts must deliver the discounted present value of lifetime differences between consumption and after-tax earnings, the realized wealth must 
be

$$
d_{t}\left(s^{t}\right)=T_{t}\left(s^{t}\right)+\mathbb{E}_{s^{t}}\left[\sum_{j=1}^{\infty} \frac{(\beta \delta)^{j} c_{t+j}\left(s^{t+j}\right)^{-\gamma}}{c_{t}\left(s^{t}\right)^{-\gamma}} T_{t+j}\left(s^{t+j}\right)\right],
$$

where $T_{t+j}\left(s^{t+j}\right) \equiv c_{t+j}\left(s^{t+j}\right)-\lambda\left(w\left(s^{t+j}\right) h_{t+j}\left(s^{t+j}\right)\right)^{1-\tau}$ is the net transfer in period $t+j$.

Given this guess for $d_{t}\left(s^{t}\right)$, it is straightforward to verify that the agent's budget constraint is satisfied (see Technical Appendix A for a complete derivation).

\section{A.2 The household model of Section 3}

Full insurance against $(g, k)$ : Assume that utility for individual $i$ in a household of $g$ adult workers and $k$ children is

$$
u\left(c, h_{i}, g, k\right)=\frac{1}{1-\gamma}\left(\frac{c}{e(g, k)}\right)^{1-\gamma}-\frac{\exp (\varphi)}{1+\sigma} h_{i}^{1+\sigma},
$$

where $c$ is household consumption and $h_{i}$ is agent $i$ 's hours worked. The household attaches equal weights to all adults and no weight to the children.

As in Section A.1, let $x_{t}=\left(a, \varphi, \alpha_{t}\right)$ denote the island-level components of the individual state. The planner can insure against realizations of $\varepsilon_{t}, g$, and $k$. The planner problem is to choose functions $c_{t}\left(x_{t}, g, k\right)$ and $h_{i t}\left(x_{t}, \varepsilon_{t}, g, k\right)$ for $i=1, \ldots, g$ to solve

$$
\max _{\left\{c_{t}\left(x_{t}, \cdot\right), h_{i t}\left(x_{t}, \cdot\right)\right\}} \int\left[\frac{g}{1-\gamma}\left(\frac{c_{t}\left(x_{t}, g, k\right)}{e(g, k)}\right)^{1-\gamma}-\sum_{i=1}^{g} \int \frac{\exp (\varphi)}{1+\sigma} h_{i t}\left(x_{t}, \varepsilon_{t}, g, k\right)^{1+\sigma} d F_{\varepsilon t}^{a}\right] d F_{t}(g, k),
$$

subject to the island-level after-tax resource constraint

$$
\int\left[c_{t}\left(x_{t}, g, k\right)-\sum_{i=1}^{g} \int \lambda\left[\exp \left(\alpha_{t}+\varepsilon_{t}\right) h_{i t}\left(x_{t}, \varepsilon_{t}, g, k\right)\right]^{1-\tau} d F_{\varepsilon t}^{a}\right] d F_{t}(g, k)=0,
$$

where eqs. (A3)-(A4) incorporate the within-island distribution $F_{t}(g, k)$ of household workers and children, and where, based on the result in Section A.1, we have already let consumption be independent of $\varepsilon_{t}$.

The first-order condition w.r.t. $c_{t}$ implies that consumption for a $(g, k)$ household is

$$
c_{t}\left(x_{t}, g, k\right)=c_{t}\left(x_{t}, 1,0\right)\left(\frac{g}{e(g, k)^{1-\gamma}}\right)^{1 / \gamma} .
$$

Combine the first-order conditions w.r.t. $c_{t}$ and $h_{i t}$ with eqs. (A4)-(A5) to derive an expression for $c_{t}\left(x_{t}, 1,0\right)$. Define $D(g, k) \equiv(\log g) / \gamma-(1-\gamma) / \gamma \log (e(g, k))$. Then use eq. (A5) and the definitions for $\widehat{\sigma}$ and $\widehat{\varphi}$ to derive the equilibrium allocations for household consumption and individual hours,

$$
\begin{aligned}
\log c_{t}\left(x_{t}, g, k\right) & =D(g, k)-(1-\tau) \widehat{\varphi}+(1-\tau)\left(\frac{1+\widehat{\sigma}}{\widehat{\sigma}+\gamma}\right) \alpha_{t}+\mathcal{C}_{t}^{a} \\
\log h_{i t}\left(x_{t}, \varepsilon_{t}, g, k\right) & =-\widehat{\varphi}+\left(\frac{1-\gamma}{\widehat{\sigma}+\gamma}\right) \alpha_{t}+\frac{1}{\widehat{\sigma}} \varepsilon_{i t}+\mathcal{H}_{t}^{a}
\end{aligned}
$$

where expressions for $C_{t}^{a}$ and $H_{t}^{a}$ are in Technical Appendix B.1. Note that hours do not depend on $(g, k)$ 
No insurance against $(g, k)$ : Consider now the model without insurance against household type. In this model, there is no within-island variation in household composition $(g, k)$. Thus the island-level components of the individual state are $x_{t}=\left(a, \varphi, \alpha_{t}, g, k\right)$, and the island planner problem corresponding to the competitive equilibrium is to choose a number $c_{t}\left(x_{t}\right)$ and functions $h_{i t}\left(x_{t}, \varepsilon_{t}\right)$ for $i=1, \ldots, g$ to solve

$$
\begin{array}{cc} 
& \max _{c_{t}\left(x_{t}\right),\left\{h_{i t}\left(x_{t}, .\right)\right.}\left\{\frac{g}{1-\gamma}\left(\frac{c_{t}\left(x_{t}\right)}{e(g, k)}\right)^{1-\gamma}-\sum_{i=1}^{g} \int \frac{\exp (\varphi)}{1+\sigma} h_{i t}\left(x_{t}, \varepsilon_{t}\right)^{1+\sigma} d F_{\varepsilon t}^{a}\right\} \\
\text { s.t. } & c_{t}\left(x_{t}\right)-\sum_{i=1}^{g} \int \lambda\left[\exp \left(\alpha_{t}+\varepsilon_{i t}\right) h_{i t}\left(x_{t}, \varepsilon_{t}\right)\right]^{1-\tau} d F_{\varepsilon t}^{a}=0 .
\end{array}
$$

In Technical Appendix B.2 we derive the following allocations:

$$
\begin{aligned}
\log c_{t}\left(x_{t}\right) & =D^{c}(g, k)-(1-\tau) \widehat{\varphi}+(1-\tau)\left(\frac{1+\widehat{\sigma}}{\widehat{\sigma}+\gamma}\right) \alpha_{t}+\mathcal{C}_{t}^{a} \\
\log h_{i t}\left(x_{t}, \varepsilon_{t}\right) & =D^{h}(g, k)-\widehat{\varphi}+\frac{1-\gamma}{\widehat{\sigma}+\gamma} \alpha_{t}+\frac{\kappa_{i t}+\theta_{i t}}{\widehat{\sigma}}+\mathcal{H}_{t}^{a},
\end{aligned}
$$

where the equivalization dummies are $D^{c}(g, k)=((1+\widehat{\sigma}) \log g-(1-\gamma) \log e(g, k)) /(\widehat{\sigma}+\gamma)$ and $D^{h}(g, k)=\left(D^{c}(g, k)-\log g\right) /(1-\tau)$.

\section{A.3 Proofs of Identification}

\section{A.3.1 Proof of Proposition 2}

The proof is organized in four recursive steps.

Step A. The four (sets of) parameters $\widehat{\sigma}, \gamma,\left\{v_{\eta t}+\Delta v_{\theta t}\right\}_{t=2}^{T},\left\{v_{\omega t}\right\}_{t=2}^{T}$ are identified from within-cohort changes in the macro moments, $\Delta \operatorname{var}_{t}^{a}(\log \hat{w}), \Delta v a r_{t}^{a}(\log \hat{h}), \Delta \operatorname{var}_{t}^{a}(\log \hat{c})$, and $\Delta \operatorname{cov}_{t}^{a}(\log \hat{w}, \log \hat{c})$, all available from $t=2, \ldots, T$. These parameters are identified recursively as follows. Each element of the sequence $\left\{v_{\omega t}\right\}_{t=2}^{T}$ is identified by:

$$
\Delta \operatorname{cov}_{t}^{a}(\log \hat{w}, \log \hat{c})^{2} / \Delta \operatorname{var}_{t}^{a}(\log \hat{c})=v_{\omega t} .
$$

Given $v_{\omega_{t}}$, each element of the sequence $\left\{v_{\eta t}+\Delta v_{\theta t}\right\}_{t=2}^{T}$ is identified by

$$
\Delta v a r_{t}^{a}(\log \hat{w})=v_{\omega t}+\left(v_{\eta t}+\Delta v_{\theta t}\right) .
$$

Given $v_{\omega t}$ and $v_{\eta t}+\Delta v_{\theta t}$, the tax-modified Frisch elasticity $\widehat{\sigma}$ is identified by

$$
\Delta \operatorname{var}_{t}^{a}(\log \hat{h})=\left[\Delta \operatorname{cov}_{t}^{a}(\log \hat{h}, \log \hat{c}) / \Delta \operatorname{cov}_{t}^{a}(\log \hat{w}, \log \hat{c})\right]^{2} v_{\omega t}+1 / \widehat{\sigma}^{2}\left(v_{\eta t}+\Delta v_{\theta t}\right) .
$$

Given $\widehat{\sigma}$, the parameter $\gamma$ is identified by

$$
\Delta \operatorname{cov}_{t}^{a}(\log \hat{h}, \log \hat{c}) / \Delta \operatorname{cov}_{t}^{a}(\log \hat{w}, \log \hat{c})=(1-\gamma) /(\widehat{\sigma}+\gamma) .
$$

Step B. Since $\widehat{\sigma}$ is known, the variances of transitory insurable shocks $\left\{v_{\theta t}\right\}_{t=1}^{T-1}$ are identified from the difference between the dispersion in growth rates ("micro moments") and the growth rate of within-cohort dispersion ("macro moments") available from $t=2, \ldots, T$ :

$\operatorname{cov}_{t}^{a}(\Delta \log \hat{w}, \Delta \log \hat{h})+\operatorname{var}_{t}^{a}(\Delta \log \hat{h})-\Delta \operatorname{cov}_{t}^{a}(\log \hat{w}, \log \hat{h})-\Delta \operatorname{var}_{t}^{a}(\log \hat{h})=2(1+\widehat{\sigma}) / \widehat{\sigma}^{2} v_{\theta, t-1}$. 
Combining the sequence $\left\{v_{\theta t}\right\}_{t=1}^{T-1}$ with $\left\{v_{\eta t}+\Delta v_{\theta t}\right\}_{t=2}^{T}$ identifies $\left\{v_{\eta t}\right\}_{t=2}^{T-1}$. Substituting the value for $v_{\theta, T-1}$ into $\left(v_{\eta T}+\Delta v_{\theta T}\right)$ from Step A identifies $\left(v_{\eta T}+v_{\theta T}\right)$.

Step C. Since $\widehat{\sigma}$ and $\gamma$ are known, the following moments, available for all $t=1, \ldots, T$ and evaluated for the youngest age group, identify the cohort effects sequence $\left\{v_{\widehat{\varphi} t}, v_{\alpha^{0} t}\right\}_{t=1}^{T}$ :

$$
\begin{aligned}
\operatorname{cov}_{t}^{0}(\log \hat{w}, \log \hat{c}) & =(1-\tau)(1+\widehat{\sigma}) /(\widehat{\sigma}+\gamma) v_{\alpha^{0} t} \\
\operatorname{cov}_{t}^{0}(\log \hat{h}, \log \hat{c}) & =(1-\tau) v_{\widehat{\varphi} t}+(1-\tau)(1+\widehat{\sigma})(1-\gamma) /(\widehat{\sigma}+\gamma)^{2} v_{\alpha^{0} t}
\end{aligned}
$$

Then $\left\{v_{\kappa^{0} t}\right\}_{t=1}^{T-1}$ and $\left(v_{\kappa^{0} T}+v_{\theta T}\right)$ are identified from

$$
\operatorname{cov}_{t}^{0}(\log \hat{w}, \log \hat{h})+\operatorname{var}_{t}^{0}(\log \hat{h})=v_{\widehat{\varphi} t}+(1-\gamma)(1+\widehat{\sigma}) /(\widehat{\sigma}+\gamma)^{2} v_{\alpha^{0} t}+(1+\widehat{\sigma}) / \widehat{\sigma}^{2}\left(v_{\kappa^{0} t}+v_{\theta t}\right) .
$$

Step D. Finally, the variances of measurement error $\left\{v_{\mu y}, v_{\mu h}, v_{\mu c}\right\}$ are identified from the following moments in levels, for example those corresponding to the youngest age group:

$$
\begin{aligned}
\operatorname{cov}_{t}^{0}(\log \hat{w}, \log \hat{h}) & =(1-\gamma) /(\widehat{\sigma}+\gamma) v_{\alpha^{0} t}+1 / \widehat{\sigma}\left(v_{\kappa^{0} t}+v_{\theta t}\right)-v_{\mu h} \\
\operatorname{var}_{t}^{0}(\log \hat{w}) & =v_{\alpha^{0} t}+\left(v_{\kappa^{0} t}+v_{\theta t}\right)+v_{\mu y}+v_{\mu h} \\
\operatorname{var}_{t}^{0}(\log \hat{c}) & =(1-\tau)^{2} v_{\widehat{\varphi} t}+(1-\tau)^{2}(1+\widehat{\sigma})^{2} /(\widehat{\sigma}+\gamma)^{2} v_{\alpha^{0} t}+v_{\mu c} .
\end{aligned}
$$

\section{A.3.2 Proof of Corollary 2.1}

At dates $t=\hat{t}, \hat{t}+1$, the data availability is the same as in Proposition 2, and hence one can identify $v_{\mu y}$. Applying Proposition 3 to dates other than $(\hat{t}, \hat{t}+1)$ when only wage and hours data are available, the whole model is then identified.

\section{A.3.3 Proof of Corollary 2.2}

From Proposition 2 we identify the parameters $\left\{\widehat{\sigma}, \gamma, v_{\mu h}, v_{\mu y}, v_{\mu c}\right\}$, the sequences $\left\{v_{\widehat{\varphi} t}, v_{\alpha^{0} t}\right\}_{t=1}^{\hat{t}}$, $\left\{v_{\kappa^{0} t}, v_{\theta t}\right\}_{t=1}^{\hat{t}-1},\left\{v_{\omega t}, v_{\eta t}\right\}_{t=2}^{\hat{t}}$, and the sums $v_{\eta, \hat{t}}+v_{\theta, \hat{t}}$ and $v_{\kappa^{0} \hat{t}}+v_{\theta \hat{t}}$.

From the cross-sectional moment $\Delta \operatorname{var}_{t}^{a}(\log \hat{c})=(1-\tau)^{2}(1+\widehat{\sigma})^{2} /(\widehat{\sigma}+\gamma)^{2} v_{\omega t}$, which is available every year, we can identify $\left\{v_{\omega t}\right\}_{t=\hat{t}+1}^{T}$. We identify the cohort effects $\left\{v_{\widehat{\varphi} t}, v_{\alpha^{0} t}\right\}_{t=\hat{t}+1}^{T}$ from the moments, available in every year,

$$
\begin{aligned}
\operatorname{cov}_{t}^{0}(\log \hat{w}, \log \hat{c}) & =(1-\tau)(1+\widehat{\sigma}) /(\widehat{\sigma}+\gamma) v_{\alpha^{0} t} \\
\operatorname{cov}_{t}^{0}(\log \hat{h}, \log \hat{c}) & =(1-\tau) v_{\widehat{\varphi} t}+(1-\tau)(1+\widehat{\sigma})(1-\gamma) /(\widehat{\sigma}+\gamma)^{2} v_{\alpha^{0} t}
\end{aligned}
$$

By combining the moments

$\operatorname{cov}_{t}^{a}\left(\Delta^{2} \log \hat{w}, \Delta^{2} \log \hat{h}\right)+\operatorname{var}_{t}^{a}\left(\Delta^{2} \log \hat{h}\right)-\Delta^{2} \operatorname{cov}_{t}^{a}(\log \hat{w}, \log \hat{h})-\Delta^{2} \operatorname{var}_{t}^{a}(\log \hat{h})=2(1+\widehat{\sigma}) / \widehat{\sigma}^{2} v_{\theta, t-2}$,

we identify $\left\{v_{\theta t}\right\}$ for the biannual years $t=\hat{t}, \hat{t}+2, \hat{t}+4, \ldots, T-2$. Note that, since $v_{\theta, \hat{t}}$ is identified, so are $v_{\eta, \hat{t}}$ and $v_{\kappa^{0} \hat{t}}$. From $\Delta^{2} v_{a r}^{a}(\log \hat{w})=v_{\omega t}+v_{\omega, t-1}+\left(v_{\eta t}+v_{\eta, t-1}+v_{\theta t}-v_{\theta, t-2}\right)$, available for $t=\hat{t}, \hat{t}+2, \ldots, T$, we can identify the sum $\left\{v_{\eta t}+v_{\eta, t-1}+\Delta^{2} v_{\theta t}\right\}$. This, together with the sequence $\left\{v_{\theta, t}\right\}$, available for $t=\hat{t}, \hat{t}+2, \ldots, T$, allows us to identify $\left\{v_{\eta t}+v_{\eta, t-1}\right\}$ for the biannual years $t=\hat{t}, \hat{t}+2, \hat{t}+4, \ldots, T-2$, as well as $\left\{v_{\eta T}+v_{\eta, T-1}+v_{\theta T}\right\}$. Finally, consider the moment

$$
\operatorname{var}_{t}^{0}(\log \hat{w})=v_{\alpha^{0} t}+\left(v_{\kappa^{0} t}+v_{\theta t}\right)+v_{\mu y}+v_{\mu h} .
$$

This moment is available for the biannual years and identifies $\left\{v_{\kappa^{0} t}\right\}$ for $t=\hat{t}, \hat{t}+2, \hat{t}+4, \ldots, T-2$ and $v_{\kappa^{0}, T}+v_{\theta T}$. 


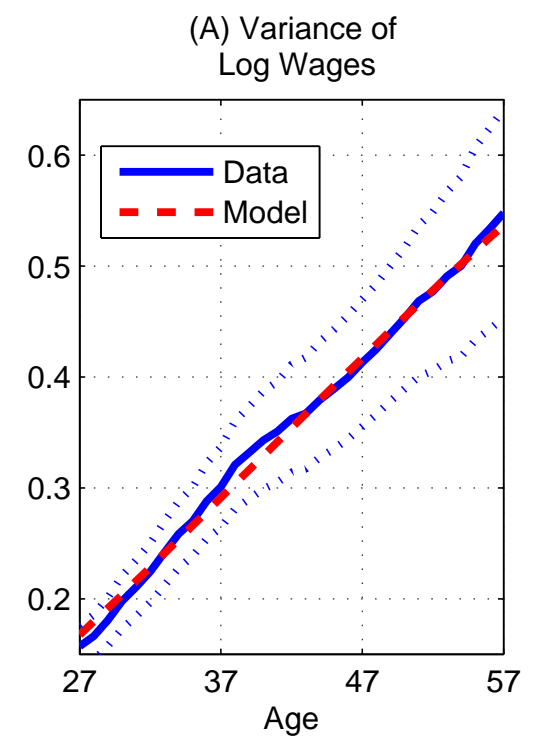

(D) Correlation between Log Wages \& Log Hours

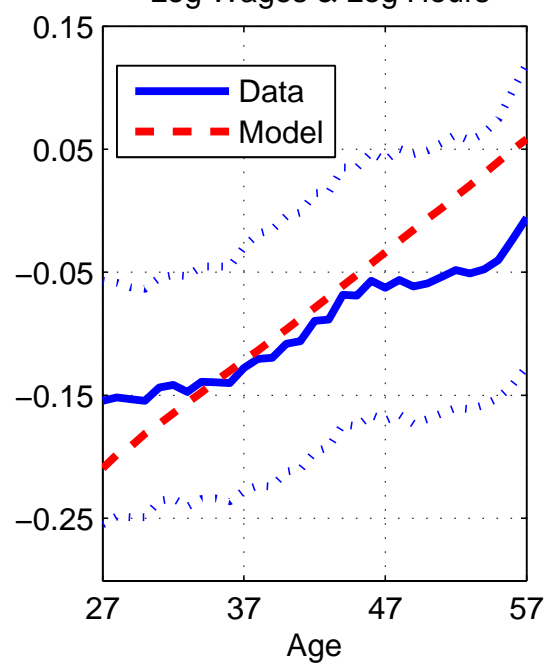

(B) Variance of Log Hours

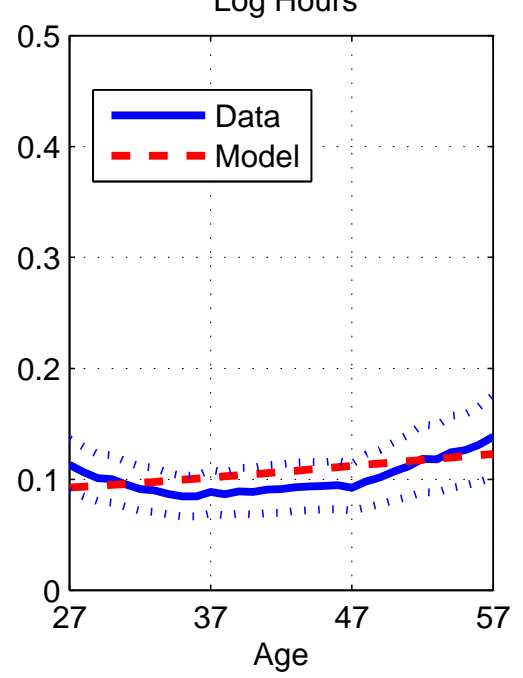

(E) Correlation between Log Wages \& Log Consumption

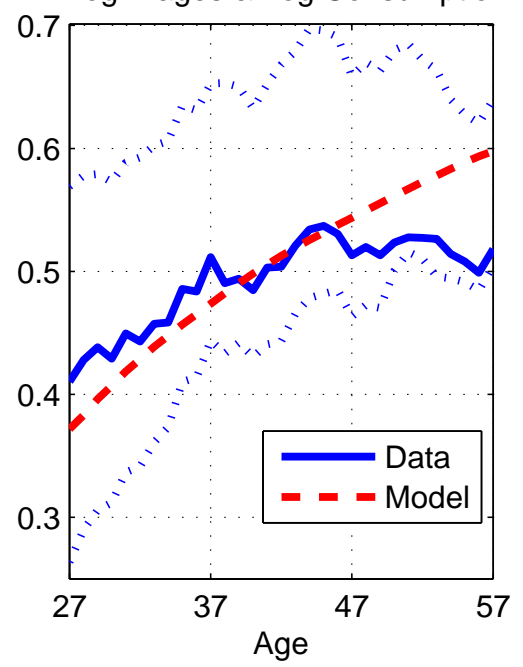

(C) Variance of Log Consumption

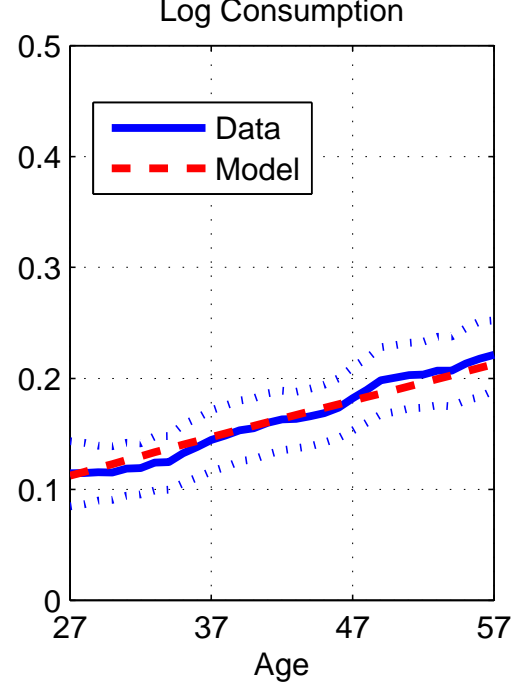

(F) Correlation between Log Hours \& Log Consumption

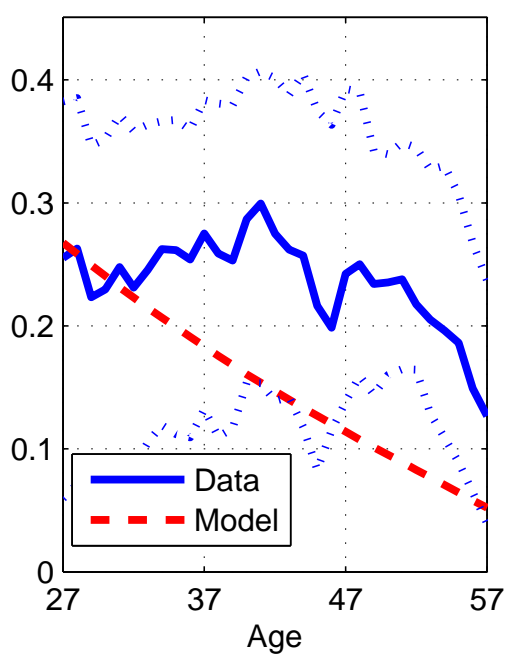

Figure 1: Data and model fit for moments in levels along the age dimension. These plots are constructed by regressing observations for all (age $a$, year $t$ ) cells on a set of age and cohort dummies. The plots show the estimated age coefficients. For the variances of wages and hours and for the wage-hours correlation, we use the entire 1967-2006 sample period. For the moments involving consumption, we use the 1980-2006 sample for which consumption data are available. The same regression procedure for constructing the age-profiles is applied to the data and to the model-generated moments. Dotted lines denote 90-10 bootstrapped confidence intervals for the empirical moments. 

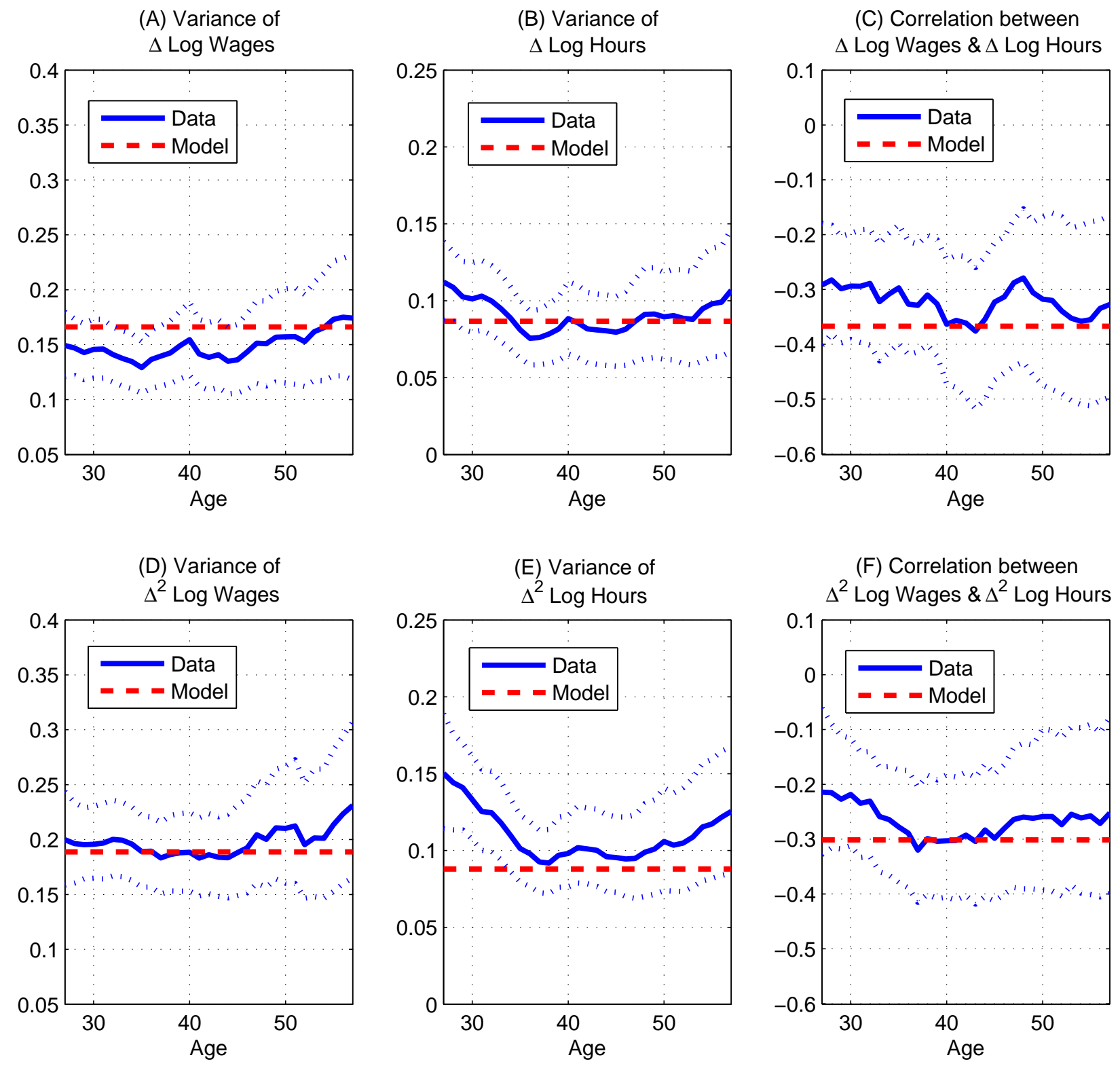

Figure 2: Data and model fit for moments in differences along the age dimension. Panels in the upper row show first differences for the years 1967-1996. Panels in the lower row show second differences for the years 1967-2006. These plots are constructed by taking the average across time for each age group $a$ : we do not control for cohort effects in constructing these plots, because differencing already eliminates cohort effects from the theoretical moments. Dotted lines denote 90-10 bootstrapped confidence intervals for the empirical moments. 

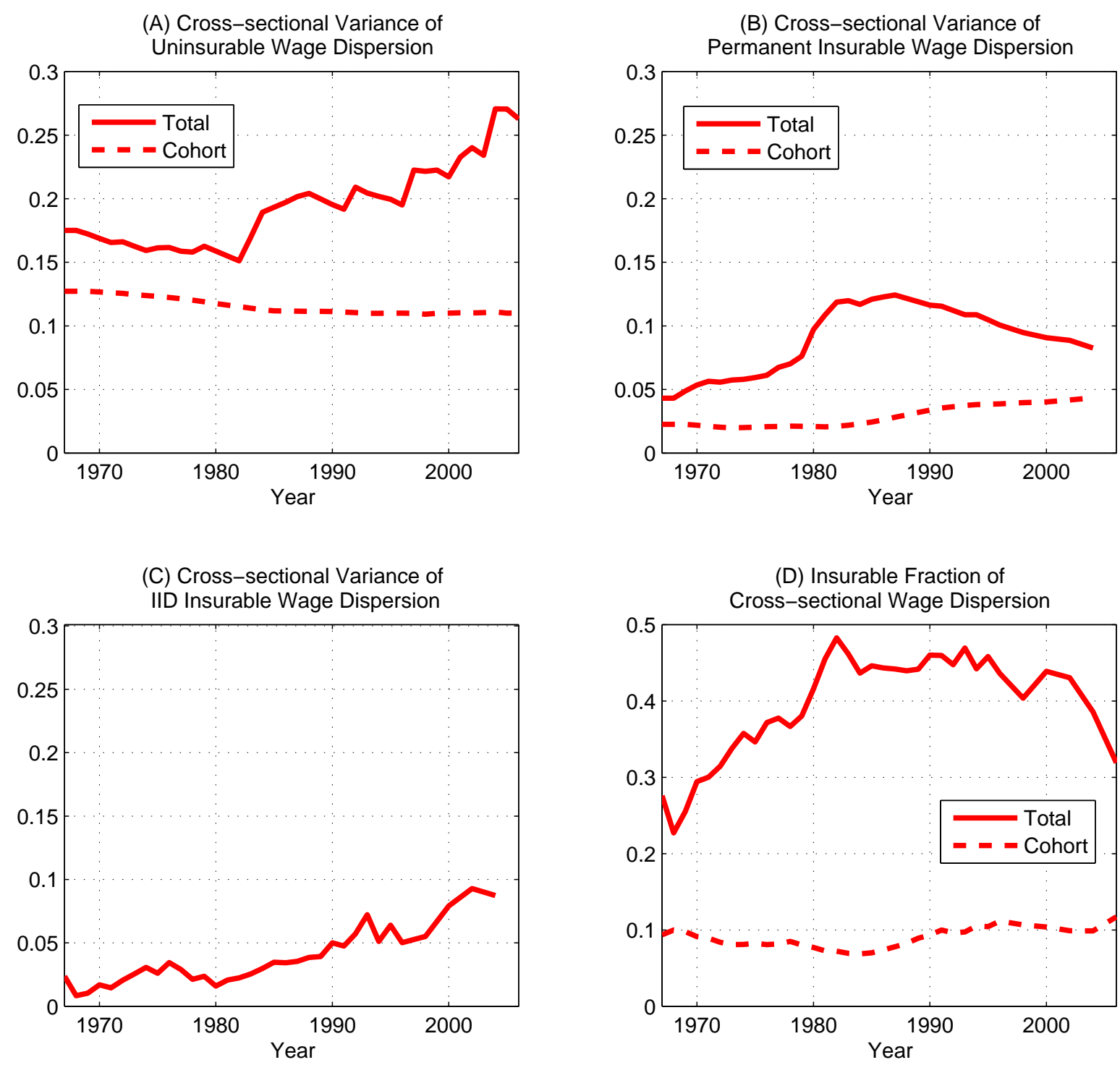

Figure 3: Panel A plots the cross-sectional variance for the uninsurable component of wages $\alpha_{t}$ (series labeled "Total") and the cross-sectional variance for the cohort-specific initial-age uninsurable component $\alpha_{t}^{0}$ (series labeled "Cohort"). Panel B plots the corresponding series for the insurable component: $\kappa_{t}$ (series labeled "Total") and $\kappa_{t}^{0}$ (series labeled "Cohort"). Panel C plots $v_{\theta t}$. In Panel D the "Total" line is the ratio of the sum of the "Total" series in Panels $\mathrm{B}$ and $\mathrm{C}$ to the total cross-sectional variance of wages (the sum of the "Total" series in Panels A, B and C). The "Cohort" line in Panel D is the ratio of the "Cohort" series in Panel B to the sum of "Cohort" lines in Panels A and B. See Table 5 in the Technical Appendix for the corresponding 90-10 bootstrapped confidence intervals. 


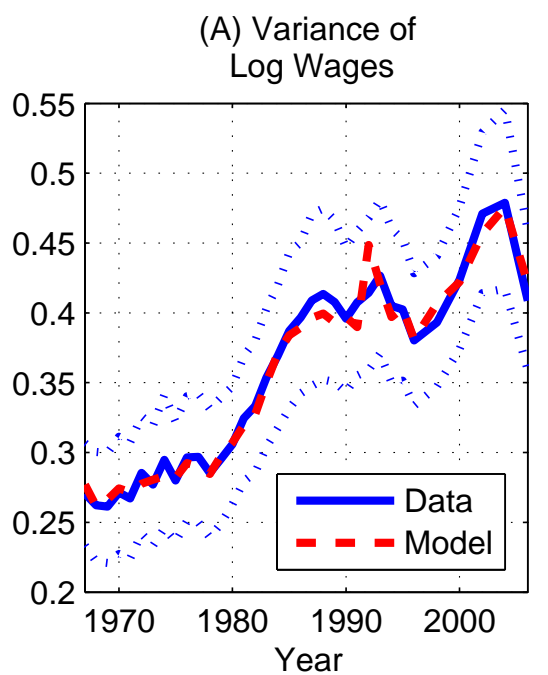

(D) Correlation between Log Wages \& Log Hours

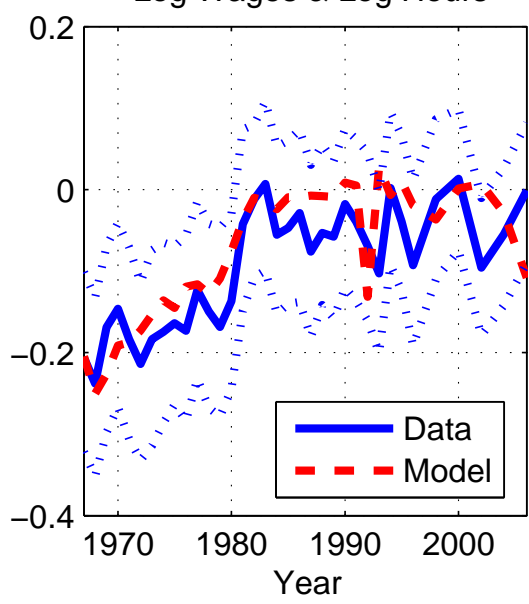

(B) Variance of Log Hours

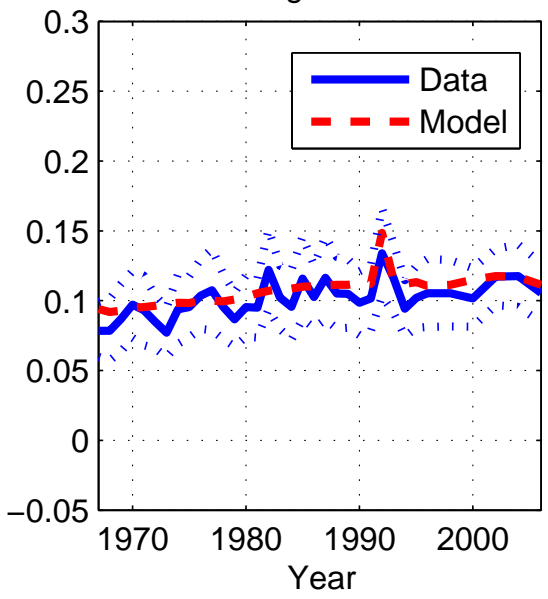

(E) Correlation between Log Wages \& Log Consumption

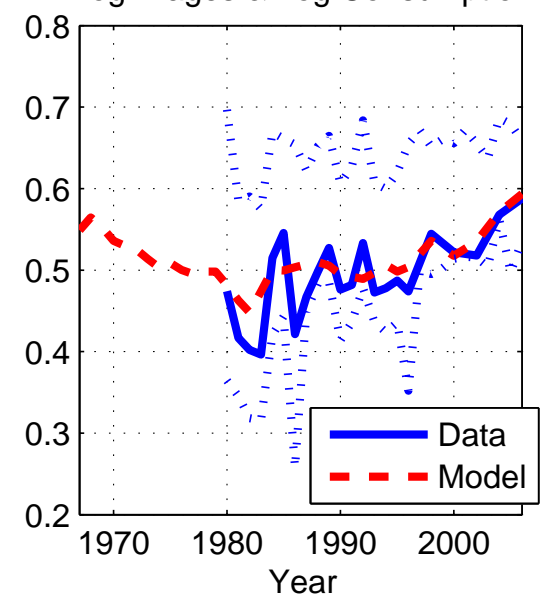

(C) Variance of Log Consumption

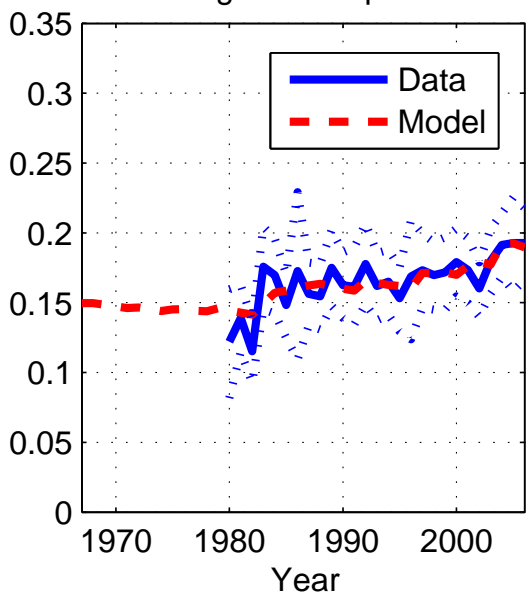

(F) Correlation between Log Hours \& Log Consumption

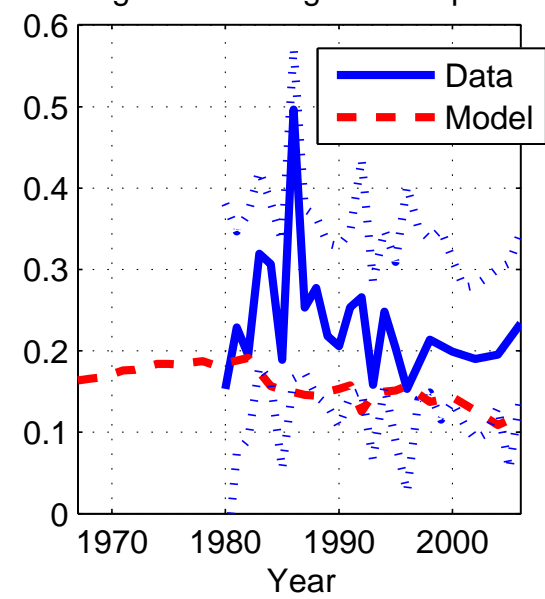

Figure 4: Data and model fit for moments in levels along the time dimension. These plots are constructed by aggregating across age groups within a given year by weighting each age group by its survival probability to account for mortality. We use the same weights in both model and data. Dotted lines denote $90-10$ bootstrapped confidence intervals for the empirical moments. 

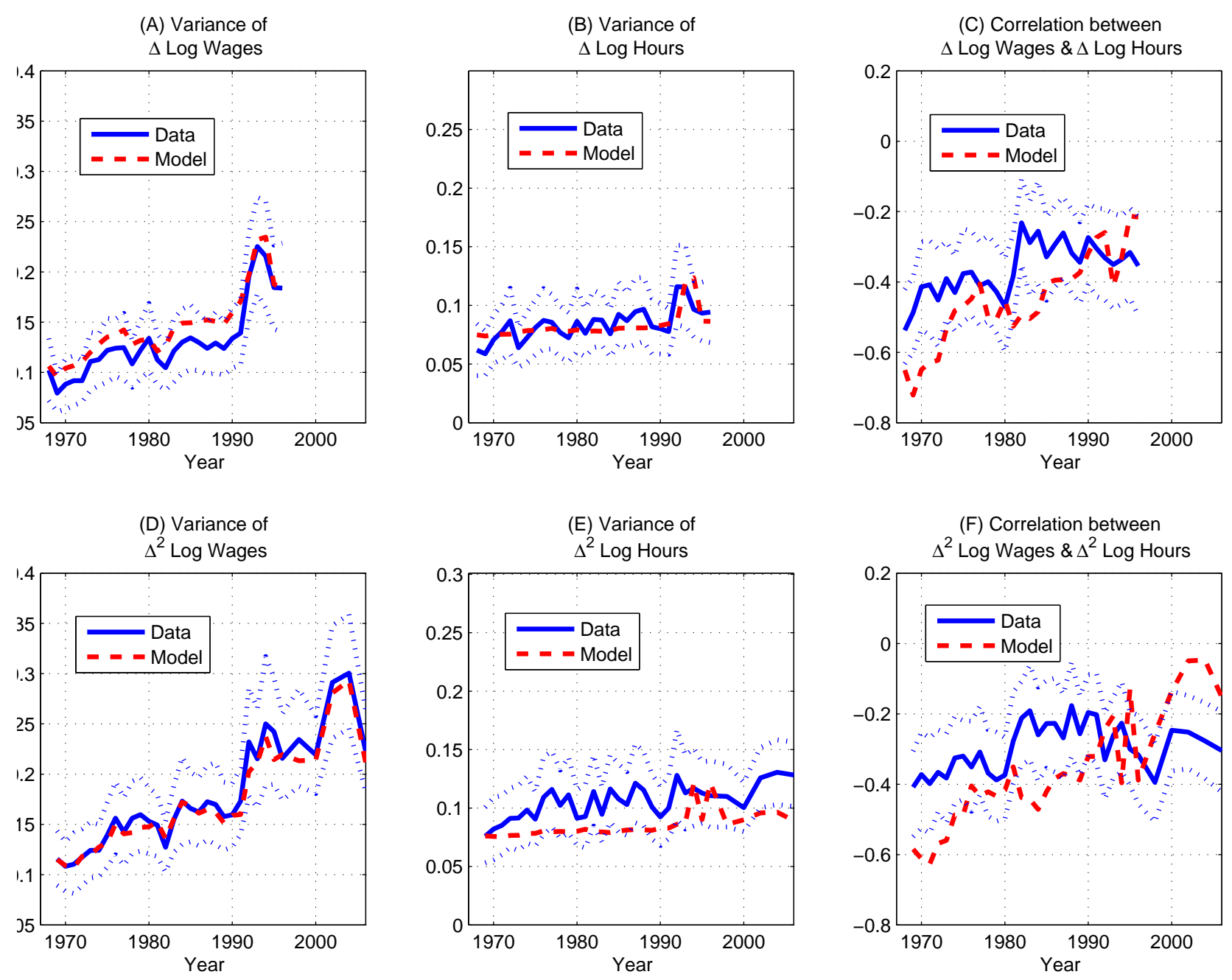

Figure 5: Data and model fit for moments in differences along the time dimension. Panels in the upper row show first differences for the years 1967-1996. Panels in the lower row show second differences for the years 1967-2006. These plots are constructed by aggregating across age groups within a given year by weighting each age group by its survival probability to account for mortality. We use the same weights in both model and data. Dotted lines denote 90-10 bootstrapped confidence intervals for the empirical moments. 
(A) Insurable Fraction of

Cross-sectional Wage Dispersion

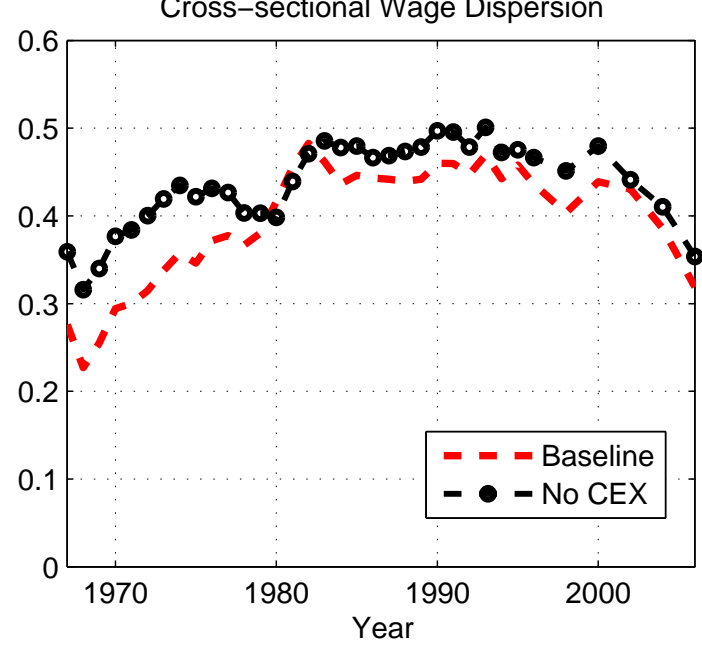

(C) Correlation between

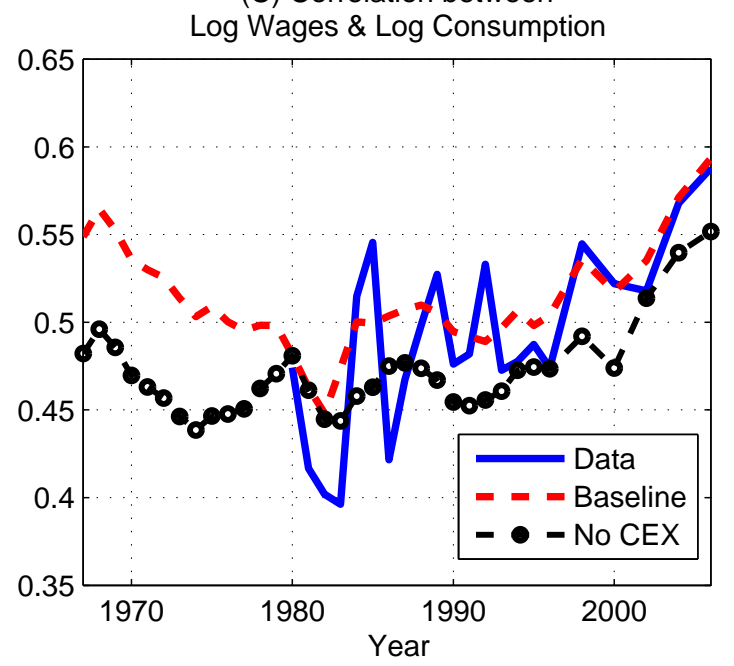

(B) Variance of

Log Consumption

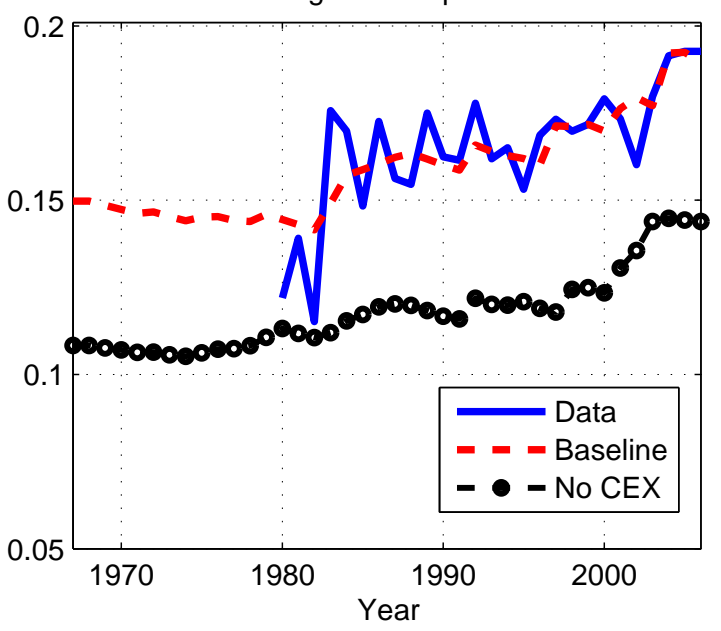

(D) Correlation between Log Hours \& Log Consumption

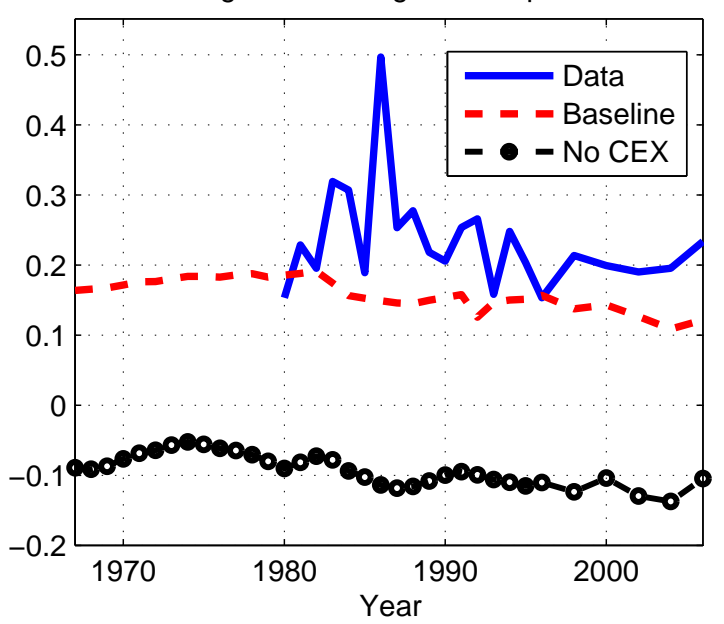

Figure 6: Data, baseline model estimated on the PSID and the CEX, and model estimated without CEX data (series labeled "No CEX"). Plots in Panel A are constructed as the line labeled "Total" in Panel A of Figure 3, and plots in Panels B-D as in Figure 4. 